\title{
Spatiotemporal Dissociation of Brain Activity Underlying Subjective Awareness, Objective Performance and Confidence
}

\author{
Qi Li, Zachary Hill, and Biyu J. He \\ National Institute of Neurological Disorders and Stroke, National Institutes of Health, Bethesda, Maryland 20892
}

\begin{abstract}
Despite intense recent research, the neural correlates of conscious visual perception remain elusive. The most established paradigm for studying brain mechanisms underlying conscious perception is to keep the physical sensory inputs constant and identify brain activities that correlate with the changing content of conscious awareness. However, such a contrast based on conscious content alone would not only reveal brain activities directly contributing to conscious perception, but also include brain activities that precede or follow it. To address this issue, we devised a paradigm whereby we collected, trial-by-trial, measures of objective performance, subjective awareness, and the confidence level of subjective awareness. Using magnetoencephalography recordings in healthy human volunteers, we dissociated brain activities underlying these different cognitive phenomena. Our results provide strong evidence that widely distributed slow cortical potentials (SCPs) correlate with subjective awareness, even after the effects of objective performance and confidence were both removed. The SCP correlate of conscious perception manifests strongly in its waveform, phase, and power. In contrast, objective performance and confidence were both contributed by relatively transient brain activity. These results shed new light on the brain mechanisms of conscious, unconscious, and metacognitive processing.
\end{abstract}

Key words: conscious perception; MEG; metacognition; slow cortical potential; subjective awareness; visual perception

\section{Introduction}

The elucidation of brain mechanisms underlying conscious perception requires distilling neural processes directly contributing to conscious awareness from those that precede, follow, or covary with it. Most investigations on brain mechanisms underlying conscious perception have adopted a "minimal contrast" approach, that is, to hold the sensory inputs as constant as possible and identify brain activities that correlate with the changing content of conscious awareness (Dehaene and Changeux, 2011). However, such a contrast between perceptual conditions would not only reveal the neural correlates of conscious perception (NCC) per se, but also include the prerequisites for (NCC-pr) and the consequences of (NCC-Co) conscious perception (Bachmann, 2009; de Graaf et al., 2012; Aru et al., 2012a). An example of the NCC-pr is the gross brain excitability or attentional state at stimulus onset that biases whether the stimulus is consciously perceived or not. Examples of the NCC-co include the rendering of verbal report and construction of long-term memory.

We aimed to address this issue by controlling for behavioral variables correlated with conscious perception. Combining lim-

\footnotetext{
Received May 1, 2013; revised Feb. 6, 2014; accepted Feb. 13, 2014.

Author contributions: Q.L. and B.J.H. designed research; Q.L. and Z.H. performed research; Q.L. and Z.H. analyzed data; Q.L. and B.J.H. wrote the paper.

This research was supported by the Intramural Research Program of the National Institutes of Health/National Institute of Neurological Disorders and Stroke. We thank Tom Holroyd, Ziad Saad, Gang Chen, John Ostuni, Ole Jensen, and Stanislas Dehaene for helpful discussions.

The authors declare no competing financial interests.

Correspondence should be addressed to Biyu J He, 10 Center Drive, Building 10, Room B1D728, Bethesda, MD 20892.E-mail: biyu.he@nih.gov.

DOI:10.1523/JNEUROSCI.1820-13.2014

Copyright $\odot 2014$ the authors $\quad 0270-6474 / 14 / 344382-14 \$ 15.00 / 0$
}

inal stimulation, forced alternative choice, and trial-by-trial introspection (Sergent et al., 2005; Wyart and Tallon-Baudry, 2008; Lamy et al., 2009; Fleming et al., 2010; Rounis and Lau, 2010; Hesselmann et al., 2011), we devised a paradigm in which we collected, on every single trial, measures of objective performance, subjective awareness, and confidence level about subjective awareness (Fig. 1A). The mutual correlations between these behavioral measures suggest that a simple experimental contrast of subjective awareness (seen vs unseen) would likely include brain activities underlying objective performance and confidence as well. However, the trial-by-trial collection of all three behavioral measures allowed us to disentangle brain activities associated with each of them. Our reasoning is as follows: since the NCC-pr affects both subjective awareness and objective performance (Lamme, 2003; Dehaene et al., 2006) while the NCC only contributes to subjective awareness (by definition), including the factor of objective performance in our design should help separate the NCC from NCC-pr. Similarly, since metacognitive processes are a component of the NCC-co (Aru et al., 2012a), including a confidence measure in our paradigm should facilitate isolating the NCC from NCC-co.

We combined this behavioral paradigm with magnetoencephalography (MEG) recording to investigate brain activities underlying conscious perception, with the correlated effects of objective performance and confidence removed. We further aimed to test the slow cortical potential (SCP) hypothesis of conscious awareness (He and Raichle, 2009b). This idea postulates that the SCPs-the low-frequency $(<4 \mathrm{~Hz})$ component of brain field potentials (He et al., 2008) - might be a correlate of conscious awareness. Previous studies have established that longlasting EPSPs at the apical dendrites of pyramidal neurons in the 
superficial layers are the major contributors to surface-recorded SCPs (Mitzdorf, 1985; Birbaumer et al., 1990; He and Raichle, 2009b). While the SCP is traditionally recorded with scalpelectroencephalography (EEG; Birbaumer et al., 1990) or surface-electrocorticography (He et al., 2008), it has recently been shown that the SCP can also be recorded using MEG (Brookes et al., 2005; Leistner et al., 2007).

\section{Materials and Methods}

\section{Subjects}

The experiment was approved by the Institutional Review Board of the National Institute of Neurological Disorders and Stroke. All subjects were right-handed and neurologically healthy with normal or correctedto-normal vision. Eleven subjects between 22 and 38 years of age (mean age 27; six females) participated in the first phase of the study consisting of a single MEG session lasting $\sim 3 \mathrm{~h}$. Two additional subjects (both female, age 24 and 25) performed three MEG sessions and one EEG session on separate days using an identical task (see below, Assessment of test-retest reliability and control for signal-to-noise ratio and EEG data collection). Last, a second cohort of 11 subjects (age range: $23-39$, mean 25 years; seven females) each participated in a session of behavioral testing with a modified version of the task (see below, Behavioral control for the length of poststimulus blank period). All subjects provided written informed consent.

\section{Stimuli and task}

The stimulus was shown on a Panasonic DLP Projector (PT-D3500U, refresh rate: $60 \mathrm{~Hz}$ ) with a $10 \%$ neutral density optical filter in front of the lens. The filter was applied to make the luminance as low as $1 \mathrm{~cd} / \mathrm{m}^{2}$ on the stimulus screen, such that every subject could reach a threshold duration longer than $16.7 \mathrm{~ms}-$ the limitation of the projector refresh rate. The stimulus was presented on a screen $75 \mathrm{~cm}$ away from the subject's eyes. Dark adaptation (at least $30 \mathrm{~min}$ ) was conducted for all 11 subjects before any behavior data were collected.

Each trial started with a white fixation cross on a gray background (Fig. $1 A)$. When the subject was ready, she/he pressed a button to start the trial. After a blank screen of a random duration between 2 and $6 \mathrm{~s}$ (following an exponential distribution), a Gabor patch ( $1^{\circ}$ visual angle/cycle) with a very low contrast $(1 \%)$ was presented for a short duration (see below). The orientation of the Gabor patch was randomly selected between 45 and $135^{\circ}$ with equal chance. Then another blank screen with a duration randomly chosen between 3 and $6 \mathrm{~s}$ (following an exponential distribution) was presented. The luminance of the blank screens was equal to the background luminance of the stimulus screen. The first blank period ensured that the subject could not predict the onset of the stimulus. The second blank period allowed enough time after stimulus offset for the analyses of slow MEG activity uncontaminated by responses to questions. Each trial ended with three sequential questions. (1) A forced alternative choice: Was the Gabor patch pointing to the upper left $\left(135^{\circ}\right)$ or upper right $\left(45^{\circ}\right)$ ?(2) Did you see the stimulus (Gabor patch) or not? (3) On a scale of 1 to 4, how confident are you about your answer to question (2), with level 4 indicating "absolutely sure" and level 1 "not sure at all." The three questions were designed to probe objective performance (OBJ), subjective awareness (SUB), and confidence level (CONF), respectively. Subjects indicated their answers to the questions via a fiber optic keypad (LumiTouch).

The experiment was conducted in two stages. In the first stage, the duration of the Gabor patch was adjusted using Levitt's staircase method (Levitt, 1971), until an individually titrated threshold for subjective awareness was found (i.e., the subject answers "seen" to question 2 in about half of the trials). The full trial as described above was used in this stage to familiarize the subject with the different questions. The distribution of threshold duration across subjects was as follows: six subjects, 33.3 $\mathrm{ms}$; three subjects, $50 \mathrm{~ms}$; two subjects, $66.7 \mathrm{~ms}$. Once the threshold duration was determined, in the second stage of the experiment, trials were shown repeatedly with identical stimulus duration at the subject's individual threshold while MEG signals were continuously recorded. Subjects performed these trials in sessions that were $<12$ min long and were allowed to rest between sessions. In total, 113-277 trials were acquired in each subject (mean \pm SD across subjects: $183.5 \pm 56.4$ ) after artifact rejection. Additionally, $6-10$ catch trials without a stimulus were shown for each subject. All of the reported behavioral and MEG results are from the second stage of the experiment only.

\section{Data acquisition}

Experiments were conducted in a whole-head 275-channel CTF MEG scanner (VSM MedTech). MEG data were collected under a sampling rate of $600 \mathrm{~Hz}$ with an anti-aliasing filter at $<150 \mathrm{~Hz}$. Before and after each recording session, the head position of the subject was measured with respect to the MEG sensor array, using coils placed on the ear canals and the bridge of the nose. All MEG data samples were corrected with respect to the refresh delay of the projector (measured with a photodiode). In 10 of 11 subjects, anatomical magnetic resonance imaging (MRI) data were acquired on a General Electric $3 \mathrm{~T}$ scanner with an 8-channel head coil, using an MP-RAGE sequence with a resolution of $1 \times 1 \times 1 \mathrm{~mm}^{3}$. The MEG data were aligned to the anatomical MRI, using the coils placed at the anatomical landmarks.

\section{Event-related fields and sensor-space ANOVA}

The Fieldtrip package (http://fieldtrip.fcdonders.nl) implemented in MATLAB (MathWorks) was used for data analysis. First, the whole recording from each session was detrended, mean removed, and bandpass filtered at $0.05-35 \mathrm{~Hz}$ with a fourth-order Butterworth filter (all filtering was done off-line with an acausal filter). Second, data were epoched from $1 \mathrm{~s}$ before the stimulus onset to $3 \mathrm{~s}$ after. Third, independent component analysis (ICA) was performed to remove eye movement and cardiac artifacts (Bell and Sejnowski, 1995), and trials with artifacts were rejected manually. Fourth, baseline correction was performed out using a window of -500 to $0 \mathrm{~ms}$.

The resultant dataset was subjected to event-related field (ERF) analysis, sensor-space and source-space ANOVA. ERF analysis consisted of averaging across trials defined as the same type (e.g., seen vs unseen). For sensor-space ANOVA, the data were downsampled to $100 \mathrm{~Hz}$ before a trial-by-trial three-way ANOVA (factors: SUB, OBJ, and CONF; dependent measure: MEG activity) was conducted at every sensor location and time point.

\section{Source localization}

Source localization was conducted using the cortically constrained L2 minimum norm estimate (MNE) implemented in Fieldtrip for the 10 subjects in whom anatomical MRI data were collected. First, brain surface for each subject was segmented and registered to the spherical atlas in FreeSurfer (http://surfer.nmr.mgh.harvard.edu/fswiki). Then the mesh points of the surface were decimated using the MNE suite (http://www.martinos.org/mne/) to obtain the same number of nodes on the cortical surface for each individual ( $N=4098$ for each hemisphere). A single-shell model was applied to each individual's cortical surface to construct the forward model.

Standard source localization methods are typically applied to trialaveraged ERFs (Dale and Sereno, 1993; Hämäläinen, 2005; but see Brookes et al., 2011). However, in our case, the correlation between different behavioral measures precludes the ERF as an accurate reflection of brain activity unequivocally underlying each behavioral measure. To resolve this issue, we constructed the source estimate for each trial individually and performed a trial-by-trial analysis in the source space. In other words, the source estimate of each trial was obtained without information from other trials, so as not to introduce correlation among behavioral factors in the source space and to allow unbiased trial-by-trial analysis. For the estimation of noise covariance matrix at the single-trial level, we used the entire time course of the full epoch ( -1 to $3 \mathrm{~s}$ ) of each trial, instead of just the prestimulus window, to achieve a more stable estimate (Brookes et al., 2011). We performed a control analysis to compare the noise covariance matrices estimated from the prestimulus window and from the full epoch, and found them to be highly similar (all $p<$ $1 \mathrm{e}-16$, assessed by spatial correlation of the half-matrix). Last, current estimates were transformed into an approximately normal distribution (using boxcox function in MATLAB; Yao and Dewald, 2005; Watthanacheewakul, 2010), and a three-way ANOVA (factors: SUB, OBJ, and 
CONF; dependent measure: source activity estimate) was performed at every source location in each subject to isolate the effect due to each behavioral measure. Source-space ANOVA was performed at selected time points from $100 \mathrm{~ms}$ to $2.5 \mathrm{~s}$ after stimulus onset.

For population analysis, results from each subject were smoothed using a $6 \mathrm{~mm}$ width kernel. The percentage of subjects showing a significant effect at each source location and time point was then calculated and subjected to binomial statistics (He and Zempel, 2013; He, 2013).

\section{Frequency-domain analyses}

For frequency-domain analyses, MEG data were epoched from $2 \mathrm{~s}$ before the stimulus onset to $3 \mathrm{~s}$ after. A longer prestimulus window was used to allow better estimation of low-frequency phase and power. For the analysis on phase, MEG data were filtered in 20 frequency bands: [0.05, 1], [1, 3], [3, 5], [5, 7] ... [37 39] Hz using a third-order Butterworth filter. Then, in each trial, the instantaneous phase was extracted from each frequency band using Hilbert transform. The phase time series from each sensor was downsampled to $50 \mathrm{~Hz}$ before a trial-by-trial two-way circular ANOVA was conducted at each time-frequency location in each subject (factors: SUB and OBJ; dependent variable: phase). A two-way instead of three-way circular ANOVA was used because statistical methods for circular data are still in development (Berens, 2009). All circular statistics were performed using the CircStat toolbox implemented in MATLAB (Berens, 2009).

For the analysis on power, a Gabor wavelet $G(t, f)=\exp \left(-\frac{t^{2}}{2 \sigma_{t}^{2}}\right)$ $\exp (j 2 \pi f t)$ with $\sigma_{t}=\frac{5}{f}$ was used. The following center frequencies were analyzed: from 2 to $30 \mathrm{~Hz}$ with $1 \mathrm{~Hz}$ steps, from 31 to $61 \mathrm{~Hz}$ with $2 \mathrm{~Hz}$ steps, and from 63 to $148 \mathrm{~Hz}$ with $5 \mathrm{~Hz}$ steps. Two hertz was the lowest frequency analyzed because accurate estimation of power requires sufficient length of data, which was limited by the length of the trial. Power time series from each trial were log-transformed into approximately normally distributed data (Wyart and Tallon-Baudry, 2008), and baseline corrected using a window of -750 to $-550 \mathrm{~ms}$. A trial-by-trial three-way ANOVA was conducted at every time frequency-sensor location in each subject (factors: SUB, OBJ, and CONF; dependent variable: MEG power). In addition, a two-sample $t$ test was performed for the effect of subjective awareness (seen vs unseen) and objective performance (correct vs incorrect), respectively.

For group analysis, significant sensors from the ANOVA (for the effect of SUB or OBJ, at a $p<0.05$ level) were pooled together by summing the $t$ score from the corresponding $t$ test across sensors $\left[\Sigma_{\mathrm{T}}\right.$ (Wyart and Tallon-Baudry, 2008) for the use of a similar metric]. To assess whether positive and negative $t$ scores might be canceled out during this process, we performed a control analysis: at each time-frequency location, sensors with positive and negative $t$ scores were summed together separately. The results from this analysis confirmed that positive and negative $t$ scores were largely separate in the time-frequency space, suggesting that cancellation effect was minimal.

The $\Sigma_{\mathrm{T}}$ metric indexes the total level of "seen vs unseen" or "correct vs incorrect" contrast across the brain. Because only significant sensors from the ANOVA were included, correlations between different behavioral measures were minimized. $\Sigma_{\mathrm{T}}$ is a normally distributed variable and was subjected to a one-sample $t$ test across the 11 subjects $\left(H_{0}: \Sigma_{\mathrm{T}}=0\right)$. Last, the population-level results were assessed for statistical significance using a nonparametric permutation test, which effectively controls the type I error (i.e., the false alarm rate) in a situation of multiple comparisons by clustering neighboring time-frequency points that exhibit the same effect (Nichols and Holmes, 2002; Jokisch and Jensen, 2007; Medendorp et al., 2007).

\section{Distribution of preferred phase in seen versus unseen trials}

This analysis was applied to the phase of the lowest frequency band $(0.05-1 \mathrm{~Hz})$ at stimulus onset only.

Cluster-level analysis. For each subject, $p$ values from the two-way circular ANOVA on phase (factors: SUB, OBJ) for the effect of subjective awareness were plotted on the scalp. In all subjects, significant sensors formed spatial clusters. We adjusted the $p$ value threshold (all $p<0.001$ ) to define at least two well-separated clusters in each subject. The two largest clusters in each subject were extracted. For each cluster thus defined, we pooled across all sensors within the cluster and all seen (or unseen) trials. A circular histogram of phase was constructed for seen and unseen trials separately, using 10 evenly distributed phase bins. The histogram shows the fraction of trials falling into each bin. The circular mean of this histogram was computed to obtain the averaged vector. The norm of this vector defined the phase-locking value (PLV) (TallonBaudry et al., 1996; Lachaux et al., 1999) and the angle of this vector defined the preferred phase. The PLV provides a direct index of the concentration of phase across trials, and the preferred phase describes the most common phase among the group of trials analyzed.

Sensor-level analysis. All sensors with a significant effect for subjective awareness (at a $p<0.001$ level) from the two-way ANOVA on phase were included in this analysis. For each sensor, the phase from each trial was described as a unit vector with the corresponding angle. This vector was circular averaged across trials of the same type (seen vs unseen) to obtain the averaged vector, the amplitude, and angle of which then defined the PLV and the preferred phase of this particular sensor.

\section{Analysis on confidence}

Because ANOVA cannot reveal brain activity monotonically related to confidence, we used an alternative method-ordinal logistic regression (De Martino et al., 2013). Confidence ranking (values: 1, 2, 3, 4) was an ordinal variable treated as the dependent measure. Independent measures included the estimated source activity (continuous variable), subjective awareness, and objective performance (binary variables). The regression model is as follows:

$$
\log \frac{P(c \leq i)}{1-P(c \leq i)}=a_{i}-\beta_{a c t} S_{a c t}-\beta_{s u b} A_{s u b}-\beta_{o b j} A_{o b j},
$$

where $c$ is the confidence level and $i$ takes values of 1,2 , and 3. $S_{\text {act }}$ is the source activity, $A_{\text {sub }}$ and $A_{\text {obj }}$ are the answers to the subjective awareness $(0$, unseen; 1 , seen) and objective performance ( 0 , incorrect; 1 , correct) questions, respectively, and $\beta_{\mathrm{act}}, \beta_{\mathrm{sub}}$, and $\beta_{\mathrm{obj}}$ are the associated regression coefficients. The left side of the above equation is called a logit function. For each logit function (associated with a particular $i$ value) there was a different intercept term $a_{\mathrm{i}}$, but the regression coefficients $\beta_{\mathrm{act}}$, $\beta_{\text {sub }}$, and $\beta_{\text {obj }}$ were the same across the three logit functions. Positive $\beta$-values indicate a positive correlation, i.e., higher confidence levels were associated with a larger predictor value. The mnrfit function in MATLAB was used to carry out the ordinal logistic regression.

For group analysis, we first computed the number of subjects showing a significant $(p<0.05) \beta_{\text {act }}$ at each source location. Only source locations with at least two subjects showing a significant result (corresponding to population $p<0.07$, binomial statistics) were analyzed further. We then computed an agreement coefficient showing the degree of agreement across subjects in terms of the sign of $\beta_{\text {act }}$. To this end, we first determined the number of subjects with a significant positive or negative $\beta_{\text {act }}$, designated Npos and Nneg, respectively. The agreement coefficient (AC) was calculated as follows:

$$
\begin{aligned}
& \text { If Npos }>\text { Nneg, } A C=N p o s /(N p o s+N n e g) ; \\
& \text { If Npos }<\text { Nneg, } A C=-N n e g /(N p o s+N n e g) ; \\
& \text { If Npos }=\text { Nneg, } A C=0 .
\end{aligned}
$$

To estimate accurately the agreement coefficient, single-subject results were not subjected to spatial smoothing in this analysis.

\section{Assessment of test-retest reliability and control for signal-to-} noise ratio

To assess test-retest reliability, we collected a substantially larger amount of data in two additional subjects (Subj. \#12 and \#13). Each of these subjects completed three experimental sessions on three different days, with each session lasting $\sim 3 \mathrm{~h}$. The task design was the same as described above. In total, 1010 and 890 trials were recorded in the two subjects (after artifact rejection), respectively. For each session, the MEG data were analyzed for sensor-space ERF and ANOVA according to procedures described above. Furthermore, to control for the unbalanced number of trials across behavioral conditions, which results in differential signal-to-noise ratio (SNR), we equated the number of trials across be- 
A

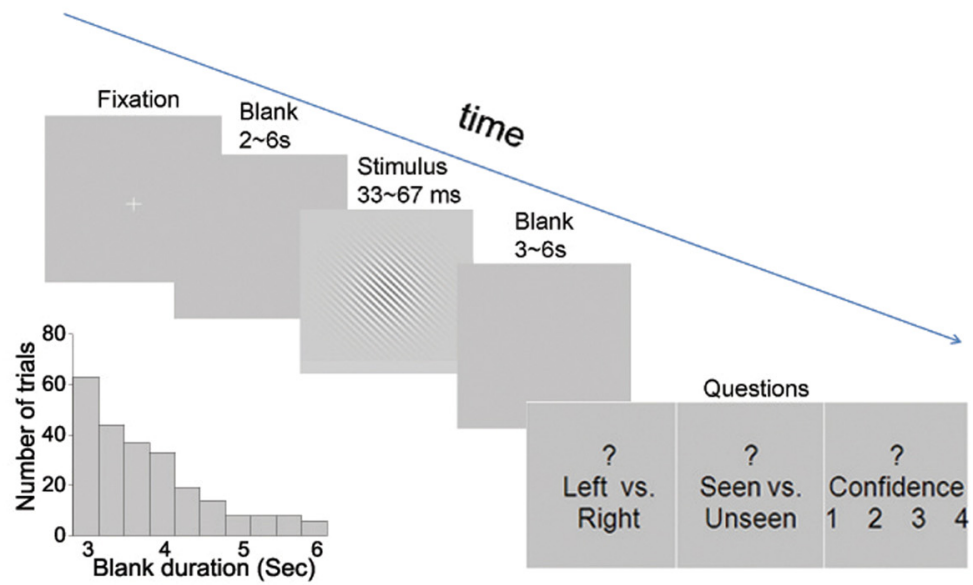

B

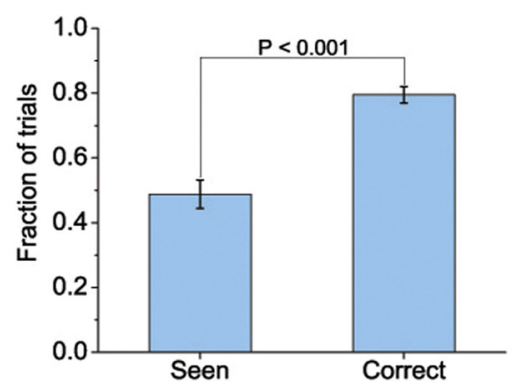

C

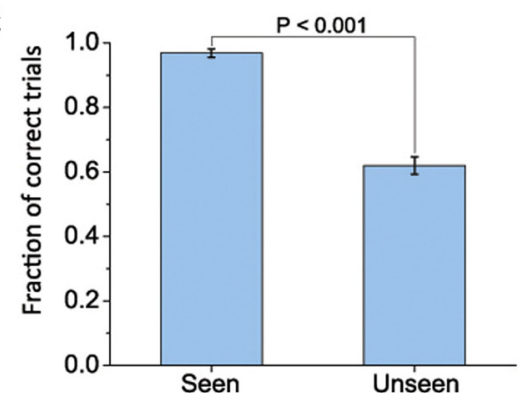

D

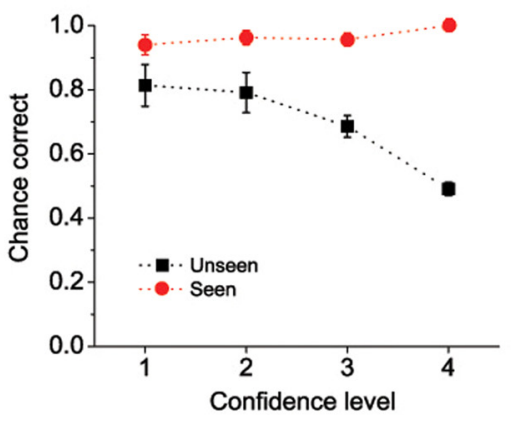

$E$

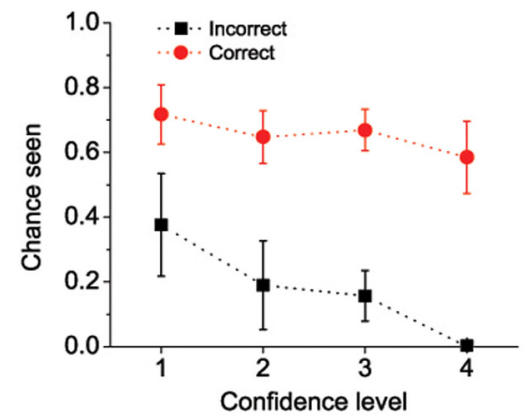

Figure 1. Task paradigm and behavioral results. $\boldsymbol{A}$, In each trial, a Gabor patch with two possible orientations $\left(45^{\circ}\right.$ or $\left.135^{\circ}\right)$ is shown for a brief duration, sandwiched between two blank screens. The duration distribution of the second blank screen from an example subject is shown in the inset. At the end of each trial, the subject is prompted to answer three sequential questions at their own pace. $\boldsymbol{B}$, The total fractions of trials in which the subjects reported seeing the stimulus (seen answers to the second question) or correctly discriminated the orientation of the Gabor patch (correct answers to the first question). $C$, The fraction of correct trials (based on orientation discrimination) in seen and unseen trials, respectively. $\boldsymbol{D}$, Objective performance (based on orientation discrimination) conditioned on subjective awareness (seen vs unseen) and confidence level. $\boldsymbol{E}$, Subjective awareness conditioned on objective performance (correct vs incorrect) and confidence level. $\boldsymbol{B}-\boldsymbol{E}$, Results are averaged across subjects. Error bars indicate SEM across subjects.

havioral conditions in an additional ERF analysis by randomly dropping out a fraction of trials (these trials were evenly distributed throughout the session). Because the head position relative to MEG sensors varied across experimental sessions, each session was analyzed separately.

\section{EEG data collection and analysis}

The same two subjects (Subj. \#12 and 13) each participated in an additional EEG session under an identical task design. EEG data were recorded using a 64-channel DC-EEG system (BrainAmpDC; Brain Products) with a mastoid reference in an EMI-shielded, sound- and lighting-controlled room. Vertical and horizontal electro-oculogram were simultaneously recorded. Analysis steps were as follows: (1) rejecting channels and data segments contaminated by artifacts via inspection of the raw data records, (2) filtering between 0.05 and $150 \mathrm{~Hz},(3)$ rereferencing to a linked-mastoid reference; (4) removal of eye blink and eye movement artifacts by an ocular correction ICA (InfoMax Extended ICA implemented in Analyzer 2; Brain Products), (5) epoching from $1 \mathrm{~s}$ before to $3 \mathrm{~s}$ after stimulus onset, (6) baseline correction using a window of -500 to $0 \mathrm{~ms}$, and (7) averaging across trials to yield ERPs. Three-hundred trials were recorded in each subject, including $10 \%$ catch trials.

\section{Behavioral control for the length of} poststimulus blank period

So far, our paradigm has used a 3-6 s poststimulus blank period to extract slow brain activity uncontaminated by the behavioral responses. To control for the potential decay of working memory during this period, we conducted behavioral testing in an additional cohort of 11 subjects using a $200 \mathrm{~ms}$ poststimulus blank period under an otherwise identical behavioral paradigm. Testing was conducted in a sound- and lighting-controlled room outside the MEG scanner; $314 \pm 48$ (mean \pm SEM) trials were recorded on each subject, including $10 \%$ catch trials.

\section{Results}

\section{Behavioral results}

The task design is illustrated in Figure $1 \mathrm{~A}$ and explained in detail in Materials and Methods (see Stimuli and task). Subjects reported seeing the stimulus in $48.9 \pm$ $4.4 \%$ (mean \pm SEM across subjects) of trials, suggesting that the threshold for subjective awareness was successfully reached. In addition, they answered correctly about the orientation of the Gabor patch in $79.5 \pm 2.5 \%$ of trials (Fig. $1 B$; chance level $=50 \%)$. When subjects reported seeing the stimulus, they correctly identified the orientation of the Gabor patch in $96.8 \pm 1.3 \%$ trials (Fig. $1 C$ ). Interestingly, even when they denied seeing the stimulus, their objective performance remained above chance level (percentage correct: $62.0 \pm 2.7 \%, p=0.001$, assessed by a Wilcoxon signed rank test against $50 \%)$. This is consistent with the "blindsight" phenomenon reported in many previous studies, i.e., even when the subject denies seeing the stimulus, their performance in a forced alternative choice question can be well above chance level (Weiskrantz, 2004; Lau and Passingham, 2006; Del Cul et al., 2009; Hesselmann et al., 2011). Since subjects' performance in orientation discrimination was much better in "seen" than "unseen" trials (Fig. 1C), objective performance and subjective awareness were indeed correlated from trial to trial.

When we considered objective performance against both subjective awareness and confidence (Fig. 1D), we found that confidence has opposite relationships with objective performance in seen versus unseen trials. In seen trials, the more confident the subject was about having seen the stimulus, the better the objective performance. In unseen trials, the more confident the subject was about not seeing the stimulus, the lower their objective performance. A two-way ANOVA indicated a significant effect of both factors and their interaction (SUB: $p<1 \mathrm{e}-15$; CONF: $p=0.002$; SUB $\times$ CONF: $p=$ $1 \mathrm{e}-5)$. Considering subjective awareness against objective perfor- 
mance and confidence (Fig. 1E), we found a significant effect of objective performance ( $p<1 \mathrm{e}-8$, assessed by a two-way ANOVA), a trend effect for confidence $(p=0.08)$, and a nonsignificant interaction effect (OBJ $\times$ CONF: $p>0.6$ ).

\section{ERFs}

We first investigated how trial-averaged ERFs vary with objective performance and subjective awareness. Separating all trials according to subjective awareness, we found that compared with unseen trials, seen trials were characterized by dramatically enhanced long-lasting ERFs starting from $\sim 300 \mathrm{~ms}$ after stimulus onset and persisting for 2-3 s, spanning the entire duration of the trial. Data from an example subject (Subj. \#1) are presented in Figure $2 A$ (top); similar qualitative results were obtained in all subjects. Importantly, this long-lasting activity is not of an oscillatory nature but rather is a slow DC-type drift- a signature of the SCP. In Subj. \#1, there was also an ERF peak at $\sim 250 \mathrm{~ms}$ that was more pronounced in seen than unseen trials; however, this peak was less reproducible across subjects. Separating all trials according to correct versus incorrect objective performance yielded similar results, although the magnitude of the long-lasting activity in correct trials was smaller than that in seen trials (Fig. $2 \mathrm{~A}$, bottom). However, because subjective awareness and objective performance were correlated from trial to trial (Fig. $1 C-E$ ), the similarity between ERFs of seen trials and correct trials could simply be due to a fraction of shared trials between them.

Indeed, when we sorted all trials according to the combination of subjective awareness and objective performance, we found that only trials that were both seen and correct still contained long-lasting ERFs (Fig. 2B; data from Subj. \#1). Unseen trials, whether correct or incorrect, did not contain long-lasting ERFs (Fig. 2B). For group analysis, we performed grand average on the absolute value of the MEG activity time course across all sensors and all subjects, for the three task conditions separately: Seen and correct; Unseen and correct; Unseen and incorrect (Fig. 2C). Because on average $96.8 \%$ of seen trials had correct objective performance, there were not enough trials in the Seen and incorrect condition for a separate ERF analysis. As can be seen in Figure $2 C$, under identical objective performance ("correct"), long-lasting MEG activity is only present in "seen" trials but not "unseen" trials. In contrast, under identical subjective awareness condition ("unseen"), there was no difference in the long-lasting activity between correct and incorrect trials. These results suggest that long-lasting MEG activity is associated with the state of subjective awareness but not objective performance. While the above results are qualitative, to quantitatively dissociate brain activities underlying subjective awareness and objective performance, and to control for the effect of confidence, we next performed a trial-by-trial factorial analysis.

\section{Trial-by-trial ANOVA on MEG activity}

A three-way ANOVA, with trial-to-trial MEG activity as the dependent variable and the three independent factors being OBJ, $\mathrm{SUB}$, and CONF, was performed on the MEG activity from every sensor in each subject. The sensor-time locations at which MEG activity correlated with subjective awareness or objective performance in three representative subjects are shown in Figure $3 A$. To present the full dataset, we used a liberal threshold here $(p<0.05$, uncorrected). Long-lasting MEG activity still correlated with subjective awareness after the effects of OBJ and CONF were both controlled for (Fig. 3A, left). In contrast, after the effects of SUB and CONF were removed, long-lasting activity no longer correlated with objective performance (Fig. $3 A$, right). Similar qualitative patterns were observed at the single-subject level in the remaining subjects. These results are in line with the ERF results reported above. Because the sign of the MEG signal depends on the geometry of the underlying dipole current flow in relation to sensor position and orientation, whether seen trials had higher activity than unseen trials in a particular sensor (Fig. $3 A$; warm colors) or vice versa (cool colors) was relatively arbitrary.

For population analysis, we compared the number of sensors showing a significant correlation to subjective awareness $(p<$ 0.05 for SUB effect, from the three-way ANOVA) with those showing a significant correlation to objective performance $(p<$ 0.05 for OBJ effect) at each time point across subjects (Fig. $3 B, C$ ). The number of sensors correlated with objective performance stayed relatively flat throughout the trial. In contrast, the number of sensors correlated with subjective awareness abruptly increased at $\sim 200 \mathrm{~ms}$; 


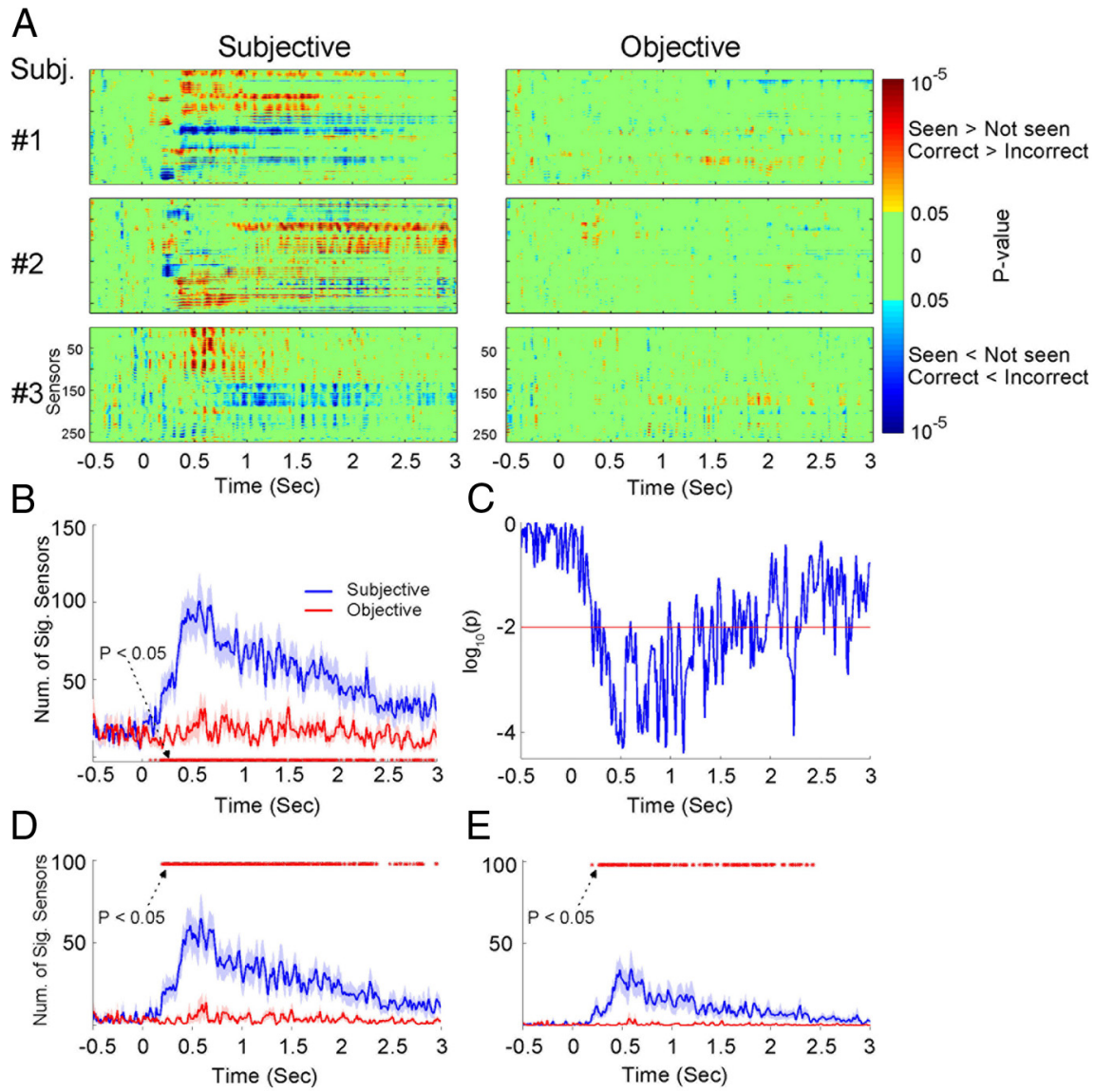

Figure 3. Trial-by-trial three-way ANOVA (factors: OBJ, SUB, and CONF) in the sensor space. $A$, Results from the ANOVA for subjective awareness (left column) and objective performance (right column), respectively. Three example subjects are shown. For each subject, the ANOVA result from each MEG sensor is shown in a separate row. Color indicates $p$ values for the effect of SUB or $O B J$, with warm colors indicating higher MEG activity in seen or correct trials, and cool colors indicating higher MEG activity in unseen or incorrect trials. $\boldsymbol{B}$, The number of significant sensors correlated with subjective awareness (blue) or objective performance (red; $p<0.05$, three-way ANOVA) averaged across all 11 subjects. Shaded areas denote SEM across subjects. Red dots indicate the time points at which the two curves significantly depart ( $p<0.05$, paired $t$ test across subjects). $\boldsymbol{C}$, The $p$ values of the paired $t$ test comparing the two curves in $\boldsymbol{B}$. Red dashed line indicates the $p=0.01$ level. $\boldsymbol{D}, \boldsymbol{E}$, As in $\boldsymbol{B}$, except that significant sensors were determined by a threshold of $p<0.01(\boldsymbol{D})$ or $p<0.001(\boldsymbol{E})$.

thereafter it reached peak at $\sim 500 \mathrm{~ms}$ and slowly returned to baseline over $2-3 \mathrm{~s}$. This result was robust to the particular threshold used for determining significant sensors $(p<0.01$, Fig. $3 D$; $p<0.001$, Fig. $3 E)$.

\section{Source localization}

To localize brain activities underlying subjective awareness and objective performance, respectively, we performed source localization on single-trial MEG data in the 10 subjects with anatomical MRI data. A three-way ANOVA (factors: OBJ, SUB, and CONF; dependent variable: estimated source activity) was performed at every source location in each subject. We focus on the effects of SUB and OBJ here, which show the estimated source activity significantly correlated with subjective awareness or objective performance, respectively, each with the other two behavioral factors controlled. Figure 4 plots the percentage of subjects at each source location and time point that showed a significant $(p<0.05)$ result for SUB or OBJ effect, thresholded at $40 \%(p<$ 0.001 , uncorrected). The highest level of overlap across subjects was $90 \%$ for subjective awareness (corresponding to $p<2 \mathrm{e}-11$ ) and $80 \%$ for objective performance (corresponding to $p<1.6 \mathrm{e}-$ $9)$. Due to the assumptions made in solving the inverse problem of MEG source modeling, statistics in the source space should be considered as "descriptive" instead of veridical.

As can be seen in Figure 4, brain activity correlated with subjective awareness or objective performance appeared in the occipital cortex at $\sim 200 \mathrm{~ms}$. It spread anteriorly into frontoparietal and temporal cortices by $\sim 300 \mathrm{~ms}$. The most pronounced difference between subjective awareness and objective performance resides between $500 \mathrm{~ms}$ and $1.5 \mathrm{~s}$, when brain activity correlated with subjective awareness covered widespread frontoparietal and temporal areas, while few source locations correlated with objective performance. By $\sim 2-2.5 \mathrm{~s}$, widespread brain activity again correlated with both subjective awareness and objective performance, likely in anticipation of the upcoming responses to questions.

\section{Phase of Slow MEG activity correlates with subjective awareness}

Thus far we have shown that long-lasting MEG activity in the time domain correlates with subjective awareness. We next investigated the frequency-domain characteristics of this activity. Inspired by earlier studies (Busch et al., 2009; Mathewson et al., 2009), we first examined whether the phase of the MEG activity correlated with subjective awareness or objective performance, each with the other behavioral factor controlled. For MEG activity from each sensor in each trial, Hilbert transform was used to extract the instantaneous phase in 20 frequency bands covering the range from 0.05 to $39 \mathrm{~Hz}$. We then performed a trialby-trial two-way circular ANOVA (factors: SUB and OBJ) on the phase of MEG activity at every time-frequency location. The results of this analysis from three representative subjects are shown in Figure 5A. At each time-frequency location, the number of sensors showing a significant ( $p<0.001$, uncorrected) effect of SUB (left) or OBJ (right) is plotted as color. Significant correlations between MEG activity phase and subjective awareness occurred in the lowest frequencies $(0.05-5 \mathrm{~Hz}$ ), with the strongest effect in the $0.05-1 \mathrm{~Hz}$ band (Fig. $5 \mathrm{~A}$, left). Much weaker correlations were found between MEG activity phase and objective performance (Fig. $5 A$, right). Similar qualitative results were obtained in all subjects.

For group analysis, we conducted a paired $t$ test across subjects on the number of sensors showing a significant correlation between MEG activity phase and subjective awareness against those showing a correlation between phase and objective performance $(p<0.001$ for the effect of SUB or OBJ, respectively, from ANOVA). Only the lowest frequencies $(<5 \mathrm{~Hz})$ had a significant difference (Fig. $5 B$ ). In particular, the $0.05-1 \mathrm{~Hz}$ band was the only frequency range showing a significant difference at and before the stimulus onset. Changing the threshold for determining significant sensors from $p<0.001$ to $p<0.01$ or $p<0.05$ yielded very similar results (data not shown). 
Sub
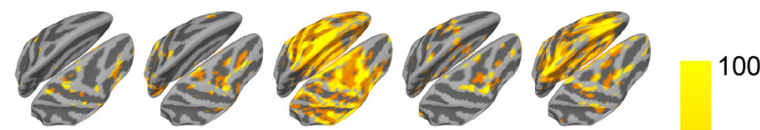

Obj $0.1 \mathrm{~s}$ $0.2 \mathrm{~s}$ $0.3 \mathrm{~s}$ $0.4 \mathrm{~s}$ $0.5 \mathrm{~s}$

bj
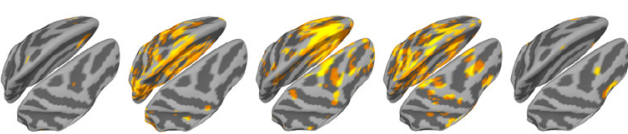

Sub
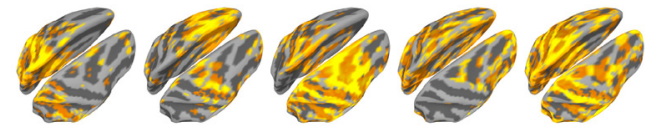

$2.5 \mathrm{~s}$

Obj
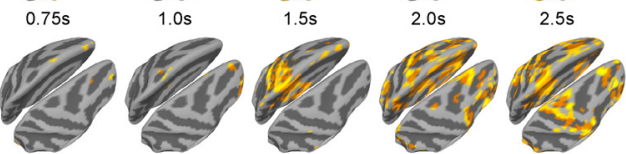

Figure 4. Trial-by-trial three-way ANOVA (factors: OBJ, SUB, and CONF) in the source space. At each source location, color indicates the percentage of subjects showing a significant ( $p<$ 0.05) effect of subjective awareness (Sub) or objective performance (Obj). Only source locations with at least 4 of 10 subjects showing a significant result are included (corresponding to population-level $p<0.001$, uncorrected). Ten time points from $100 \mathrm{~ms}$ to $2.5 \mathrm{~s}$ after stimulus onset are shown.

\section{A}
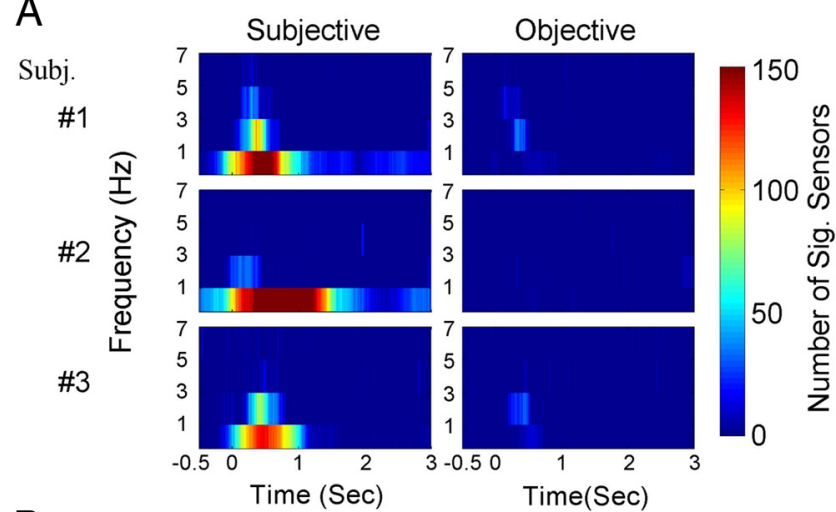

B

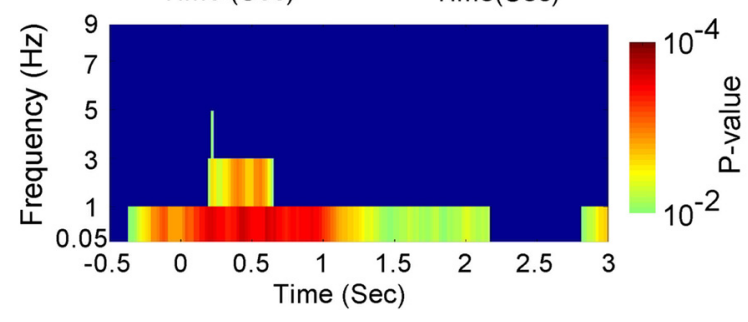

Figure 5. Trial-by-trial two-way circular ANOVA (factors: OBJ and SUB) on phase in the sensor space. $\boldsymbol{A}$, Results from the ANOVA for subjective awareness (left column) and objective performance (right column), respectively. Results from three example subjects are shown. Color plots the number of significant $(p<0.001$ ) sensors at each time-frequency location. $\boldsymbol{B}, \boldsymbol{A}$ paired $t$ test across all 11 subjects was performed at each time-frequency location on the number of sensors showing a significant $(p<0.001)$ SUB versus OBJ effect. The $p$ values are plotted as color and thresholded at $p<0.01$.

The distribution of SCP phase in seen versus unseen trials The above results show that the phase of the SCP correlates with subjective awareness even after the effect of objective performance was removed. This raises the following question: At which phase(s) of the SCP is stimulus presentation more likely to be consciously perceived? To answer this question, we focused on the phase of $0.05-1 \mathrm{~Hz}$ activity at stimulus onset. Figure $6 \mathrm{~A}$ shows MEG sensor locations with a significant correlation between phase and subjective awareness (assessed by the ANOVA) in an example subject (Subj. \#1). It can be seen that significant sensors formed spatial clusters. For the two largest clusters, the distribution of phase across seen and unseen trials are shown in Figure $6 B$. While the phase in unseen trials was distributed uniformly around the circle (blue lines), in seen trials it concentrated around $\frac{\pi}{4}$ and $-\frac{3 \pi}{4}$ in the two clusters, respectively. Phase $\frac{\pi}{4}$ and $-\frac{3 \pi}{4}$ are opposite phases to each other, suggesting that the underlying dipole current flow is likely located between these two clusters of sensors in a perpendicular direction.

We next extracted two dominant clusters of sensors based on the correlation between phase $(0.05-1 \mathrm{~Hz}$ at stimulus onset) and subjective awareness for each subject in a similar manner (number of sensors included in each cluster: $12.8 \pm 2.8$ ), and calculated their phase histograms for seen and unseen trials separately. From the phase histogram we obtained two metrics: (1) a PLV describing the degree of phase concentration across trials, with value 0 indicating a uniform distribution and value 1 indicating identical phase value across trials and (2) a preferred phase describing the most commonly observed phase. For the 22 clusters, PLV is plotted against the preferred phase for seen (red) and unseen (blue) trials, respectively (Fig. 6C). The degree of phase concentration was significantly higher in seen trials than unseen trials (paired $t$ test on PLV across clusters, $p<1.6 \mathrm{e}-12$ ). Moreover, in unseen trials, the distribution of preferred phase across the 22 clusters did not depart from uniformity (Rao's test for circular uniformity, $p>0.5$ ), whereas the preferred phase in seen trials followed a bimodal distribution, concentrating around $\frac{\pi}{4}$ and $-\frac{3 \pi}{4}$ (departure from uniformity: $p<0.01$, Rao's test).

To ensure that the above results did not depend on our choice of clusters, we additionally performed a sensor-level analysis. All sensors from all subjects with a significant correlation between phase and subjective awareness ( $p<0.001$, assessed by ANOVA) were included in this analysis. We obtained the PLV and preferred phase for each sensor in seen and unseen trials, respectively (Fig. 6D). Across 635 sensors, the preferred phase in seen trials again clustered around $\frac{\pi}{4}$ and $-\frac{3 \pi}{4}$. The degree of phase concentration was dramatically higher in seen trials than unseen trials (paired $t$ test on PLV across sensors, $p<2.6 \mathrm{e}-298$ ).

\section{Power of MEG activity}

To investigate the relationship between the power of MEG activity and subjective awareness or objective performance, a threeway ANOVA (factors: SUB, OBJ, and CONF) was performed on MEG activity power from each sensor at every time-frequency location. In addition to the ANOVA, we also performed a twosample $t$ test on power between seen and unseen trials, and between correct and incorrect trials. Because our previous analyses revealed widespread activity associated with subjective awareness (Figs. 3, 4), for group analysis we adopted a whole-brain instead of region of interest approach. For subjective awareness and objective performance, the $t$ score was summed across sensors showing a significant ANOVA result (at a $p<0.05$ level for SUB and OBJ, respectively, for details see Materials and Methods). We then assessed whether this summed $t$ score $\left(\Sigma_{\mathrm{T}}\right)$ was significant across the 11 subjects (one-sample $t$ test against 0 , corrected for multiple comparisons using cluster-based nonparametric permutation test). The results of this analysis are shown in Figure 7. For the effect of subjective awareness (Fig. 7, left), we found that 


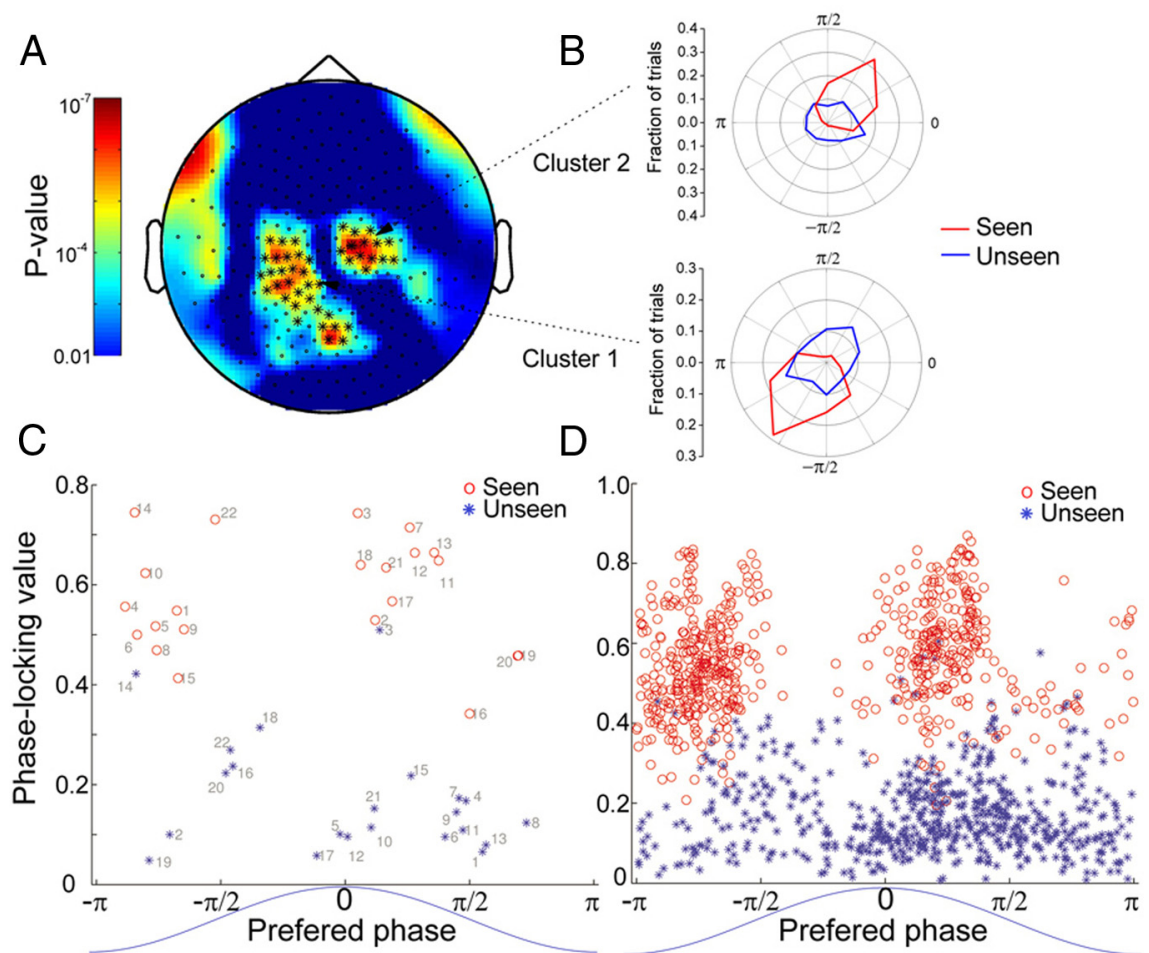

Figure 6. Phase of low-frequency MEG activity in seen versus unseen trials. $A$, Scalp plot of $p$ values for the effect of subjective awareness determined by the two-way ANOVA on phase (0.05-1 Hzactivity at stimulus onset), from an example subject (Subj.\#1). Two dominant sensor clusters are defined by the contour of $p$ values ( $\left.{ }^{*} p<0.0001\right)$. $\boldsymbol{B}$, The phase histograms for seen (red) and unseen (blue) trials for the two dominant clusters in $A$. C, PLV is plotted against preferred phase for the two dominant clusters from each subject, calculated for seen (red) and unseen (blue) trials separately. The numbers indicate the cluster number (from 1 to 22 ). $D$, PLV and preferred phase for all significant ( $p<0.001$, from ANOVA result for SUB effect) sensors in seen (red) and unseen (blue) trials, respectively. The blue sinusoidal curves at the bottom of $C$ and $\boldsymbol{D}$ show that phase 0 relates to the peak of the fluctuation, and phase $\pm \pi$ to the trough of the fluctuation.

MEG power in alpha, beta, and low gamma frequency ranges $(\sim 10-50 \mathrm{~Hz})$ was lower in seen compared with unseen trials, in a window of $\sim 500 \mathrm{~ms}$ to $1 \mathrm{~s}$ (cluster-level $p<0.001$ ). In contrast, MEG power in the lowest frequencies $(2-6 \mathrm{~Hz})$ was significantly higher in seen trials than unseen trials during a $500 \mathrm{~ms}$ window following stimulus onset $(p<0.001)$. No significant result was observed in the high gamma frequency range. The correlation between MEG power and objective performance in the low-mid frequency ranges $(<30 \mathrm{~Hz})$ was qualitatively similar but much weaker (Fig. 7, right), with decreased power in alpha and beta frequency ranges $(p<0.008)$ and increased power in the $2 \mathrm{~Hz}$ band $(p<0.02)$ in correct compared with incorrect trials. In addition, correct trials were associated with increased power in the high gamma frequency range $(p<0.008)$.

\section{MEG activity correlated with confidence}

Thus far we have focused on extracting brain activities underlying subjective awareness and objective performance while controlling for confidence as a covariate. Last, we investigated brain activities contributing to confidence. While subjective awareness and objective performance are both binary variables, confidence measure in our paradigm is an ordinal variable with four levels. Because ANOVA cannot extract MEG activity correlated with confidence in a monotonic manner, we adopted an alternative approach. After source localization, trial-by-trial ordinal regression (De Martino et al., 2013) was performed at each source location to identify MEG activity monotonically correlated with confidence, with the effects of subjective awareness and objective performance both controlled. The results of this analysis are shown in Figure 8. Source activity correlated with confidence first appeared in the dorsal parietal cortex at $\sim 200 \mathrm{~ms}$. It then moved anteriorly and had widespread frontoparietal distribution at $\sim 500 \mathrm{~ms}$. The activity was restricted to posterior brain regions at $\sim 750$ ms and dissipated thereafter. It reappeared around central and frontal cortices at 2-2.5 s, possibly in anticipation of the upcoming responses to questions. These results show that compared with subjective awareness (Fig. 4), confidence is associated with relatively transient MEG activity.

\section{Test-retest reliability and control for} SNR across behavioral conditions Additional MEG data were collected for two control analyses. First, to assess testretest reliability, we obtained three experimental sessions on different days in two additional subjects (Subj. \#12 and \#13). A three-way ANOVA (factors: SUB, OBJ, and CONF) was performed on data from each session in the sensor space. Similar qualitative results were obtained across days in both subjects: namely, that longlasting MEG activity correlated with subjective awareness but not objective performance (Fig. 9A). Because the sensor locations in relation to the brain varied across sessions, the spatial patterns of the activity were variable.

Second, because different behavioral conditions are associated with different numbers of trials (e.g., the three conditions in Fig. $2 B$ contained 58, 32, and 24 trials, respectively), the differential SNR across conditions might confound our results. To address this issue, in Subj. \#12 and \#13, we conducted a control analysis by using an identical number of trials in each condition to compute the ERF. Results from a representative session in each subject are shown in Figure $9 B$. These results are similar to those shown in Figure $2 B$ : long-lasting MEG activity was observed only in Seen and correct condition, but not in Unseen and correct or Unseen and incorrect conditions. Thus, after controlling for SNR, we confirmed that slow MEG activity correlates with subjective awareness but not objective performance.

\section{Slow EEG activity correlates with subjective awareness}

Previous EEG studies have established that the negative shift of scalp-recorded SCP indexes increased cortical excitability (Rockstroh et al., 1989; Birbaumer et al., 1990). Because the polarity of MEG signals depends on the position and direction of the underlying dipole current flow in relation to the MEG sensor, there is no unique relationship between MEG signal polarity and changes in cortical excitability. This is consistent with our finding that both positive and negative going slow MEG activities correlated with subjective awareness (Figs. 2, 3). To further elucidate the underlying cortical excitability state associated with subjective awareness, we performed a preliminary EEG study. The same two subjects as used in the additional MEG data collection (Subj. \#12 and \#13) each performed an EEG session under the same task 


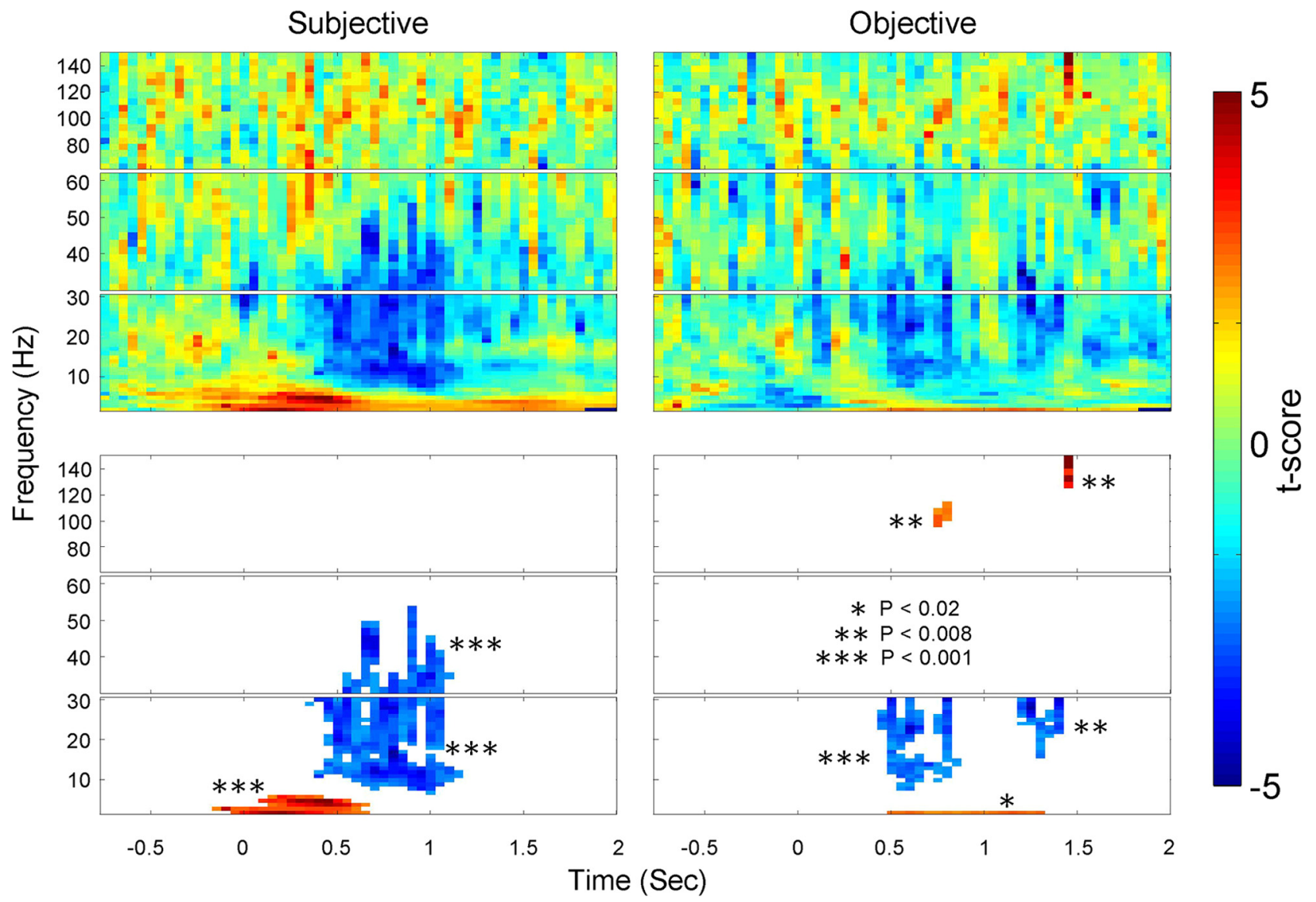

Figure 7. Group analysis results on MEG power for the effect of subjective awareness (left) and objective performance (right). For each subject and at each time-frequency location, the $t$ scores from a two-sample $t$ test (seen vs unseen, or correct vs incorrect) were summed across significant sensors as determined by the ANOVA ( $\Sigma_{T}$, see Materials and Methods). The $\Sigma_{T}$ value was subjected to a population test across subjects (one-sample $t$ test against 0 ), and the resulting t score is plotted as color (top). Warm colors indicate that power was higher in seen (left) or correct (right) trials; cool colors indicate that power was higher in unseen or incorrect trials. Bottom, Significant time-frequency clusters after controlling for multiple comparisons using a nonparametric permutation test. The statistical significance of each cluster is indicated in the graph; color is the same as in top, indicating the population-test $t$ score.

paradigm. We sorted all trials into three categories as in Figures $2 B$ and $9 B-$ Seen and correct, Unseen and correct, Unseen and incorrect, and computed ERPs for each condition. The results are shown in Figure 10. In the Seen and correct condition, we observed a transient negative potential at $\sim 300 \mathrm{~ms}$ followed by longlasting positive EEG potential that peaked around $500 \mathrm{~ms}$ and returned to baseline around $1.5 \mathrm{~s}$. The transient negative potential was distributed posteriorly, corresponding to the initial feedforward excitation in the early and higher order visual cortices. The late positive potential had a central parietal distribution, consistent with earlier studies (Dehaene and Changeux, 2011). This slow positive EEG potential suggests that it was profound cortical inhibition, instead of excitation, that correlated with subjective awareness of the stimulus.

Behavioral control for working memory decay

So far our paradigm has used a relatively long poststimulus blank period (3-6s) to elucidate slow brain activity uncontaminated by behavioral responses. While this design is similar to several pre-
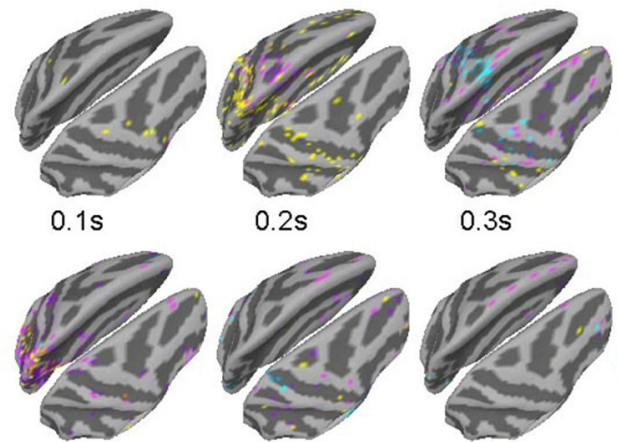

$1.0 \mathrm{~s}$

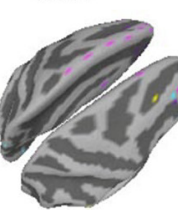

$1.5 \mathrm{~s}$

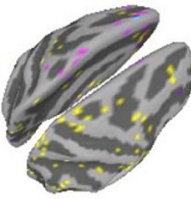

$0.4 \mathrm{~s}$

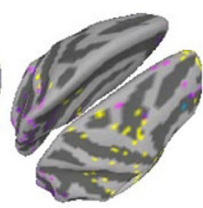

$2.0 \mathrm{~s}$

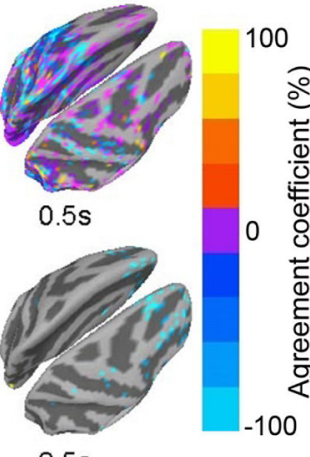

Figure 8. MEG activity correlated to confidence in the source space. At each source location, the relationship between estimated source activity and confidence level was evaluated by ordinal regression (covariates: SUB and OBJ). Only source locations with at least two subjects showing a significant result were included. The agreement coefficient (see Materials and Methods) is plotted as color at different poststimulus time points. 
A

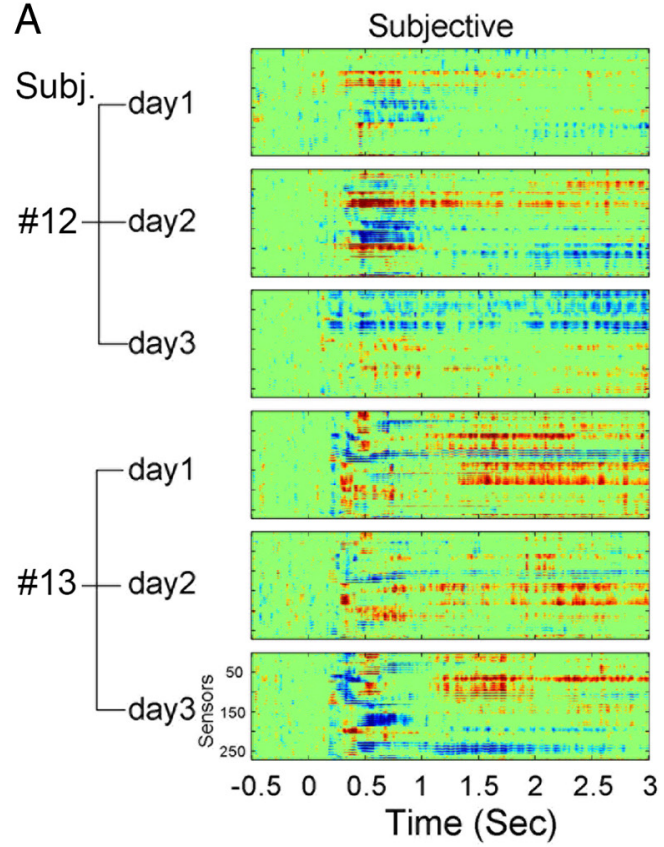

B

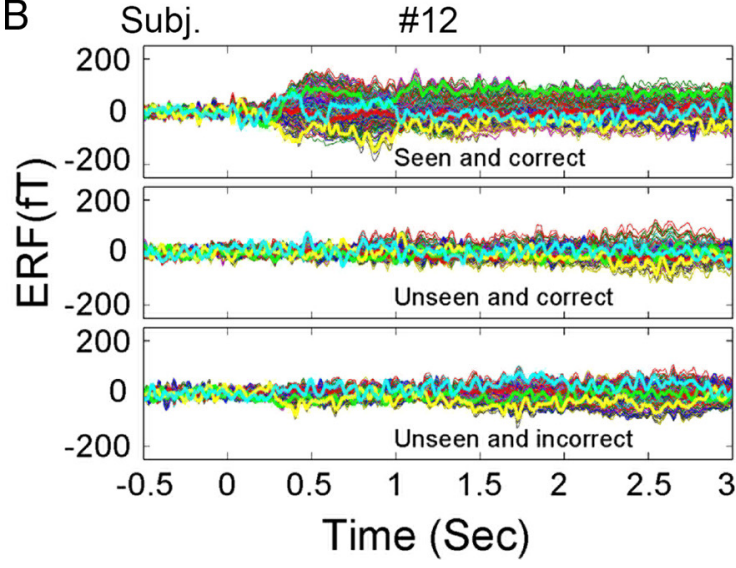

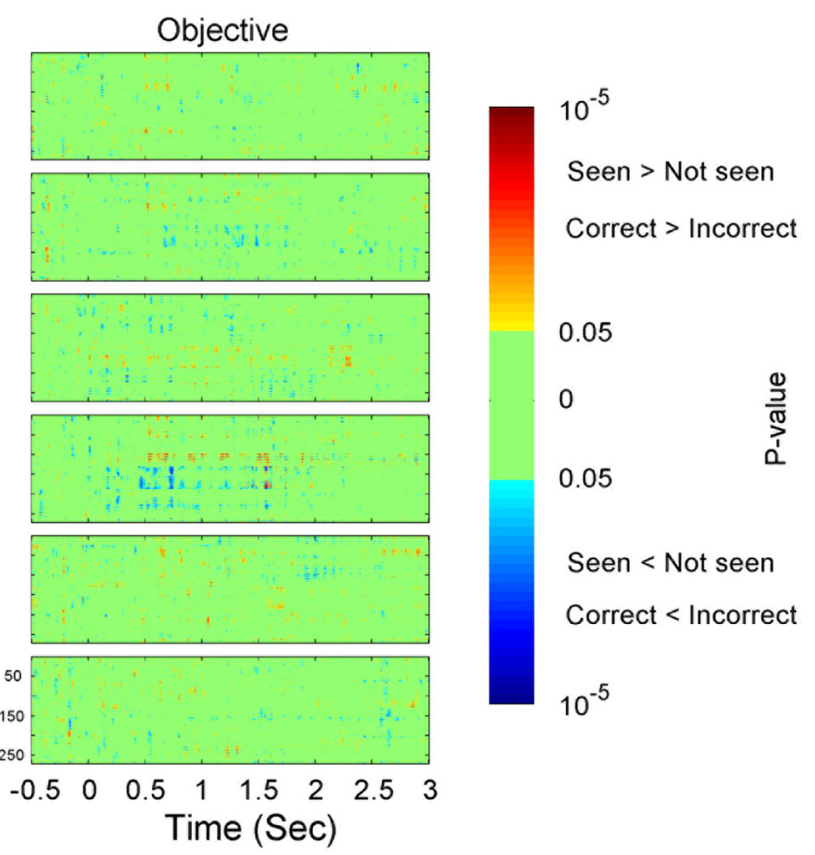

\#13

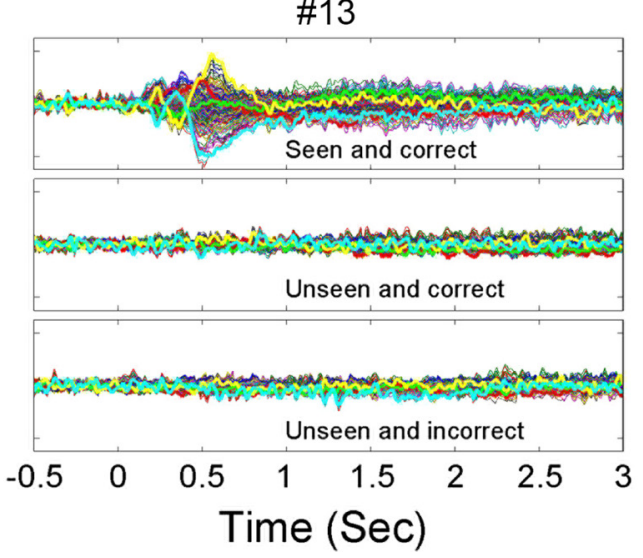

Figure 9. Test-retest reliability assessment and control for SNR. A, Sensor-space three-way ANOVA (factors: SUB, OBJ, and CONF) was performed for each recording session in Subj. \#12 and \#13. The effect of SUB and OBJ are shown in the left and right columns, respectively. Format is the same as in Figure 3A. B, The ERFs from a representative session in each subject (day 2 in Subj. \#12; day3 in Subj. \#13) for three conditions separately: Seen and correct, Unseen and correct, and Unseen and incorrect. The number of trials was equated across the three conditions by dropping out a random fraction of trials, resulting in 84 trials per condition in Subj. \#12 and 76 trials per condition in Subj. \#13. Format is the same as in Figure $2 B$.

same as reported in Fig. 1), both analyzed according to signal detection theory (Green and Swets, 1966; Fig. 11).

For subjective awareness, the detection $d^{\prime}$ was highly significant for both long- and short-duration tasks $(p=5 \mathrm{e}-7$ and 0.0004; Fig. 11A). Moreover, subjects adopted a conservative criterion in both tasks, as reflected in the criterion being significantly above $0(p=8 \mathrm{e}-7$ and 0.0008 ; Fig. $11 A)$. For objective performance, discrimination $d^{\prime}$ was also highly significant for both long- and short-duration tasks ( $p=8 \mathrm{e}-6$ and $5 \mathrm{e}-5$ ), while criterion was close to 0 , indicating very small biases (Fig. 11B). A two-way repeated-measures ANOVA on $d^{\prime}$ [between-subject factor: task (long vs short duration); within-subject factor: behavior (subjective awareness/detection vs objective performance/discrimination)] revealed a significant effect for task $(p<0.01)$ with higher $d^{\prime}$ in the long-duration task, while the effect of behavior and the interaction of duration $\times$ behavior were not significant. The higher $d^{\prime}$ during the long-duration task could be due to the smaller number of catch trials included therein, or better conscious/postconscious processing allowed by the longer post- stimulus blank duration. A similar two-way ANOVA on criterion revealed a nonsignificant effect of task.

We next considered the discrimination $d^{\prime}$ for objective performance against subjective awareness and confidence and found a similar pattern across both tasks (Fig. 11C). The effect of subjective awareness was highly significant (Long duration: $p=4 \mathrm{e}-15$; Short duration: $p=3 e-9)$, and the interaction of subjective awareness and confidence was significant (Long duration: $p=0.01$; Short duration: $p=0.0006)$, while confidence by itself was not significant in either task $(p>0.7)$. Altogether, these results suggest that the behavioral results we obtained under MEG recording were qualitatively similar to those obtained in the behavioral control experiment with a much shorter poststimulus blank period (200 ms vs 3-6 s) and therefore were unlikely to be significantly affected by potential working memory decay during the poststimulus period.

\section{Discussion}

In summary, we found that long-lasting, low-frequency MEG activity correlates with the state of subjective awareness, even 
A Subj \#12

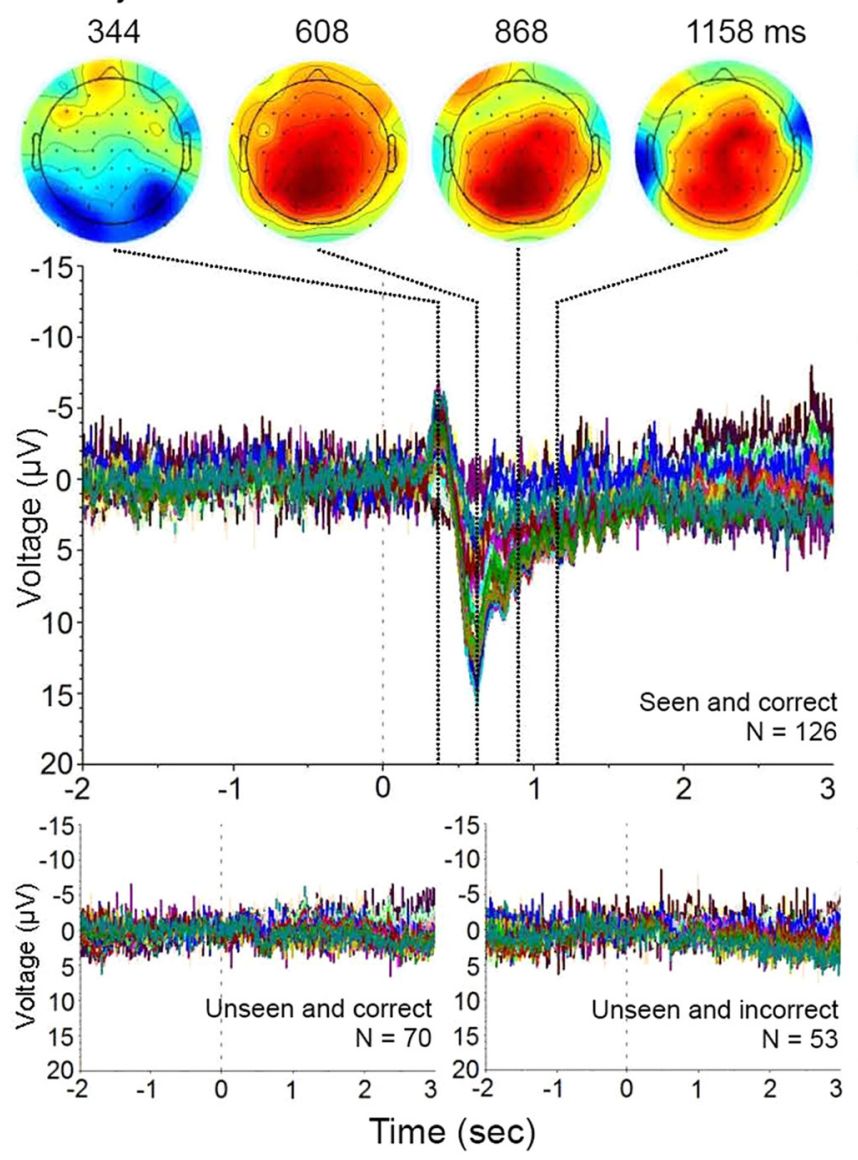

B Subj \#13 386

596
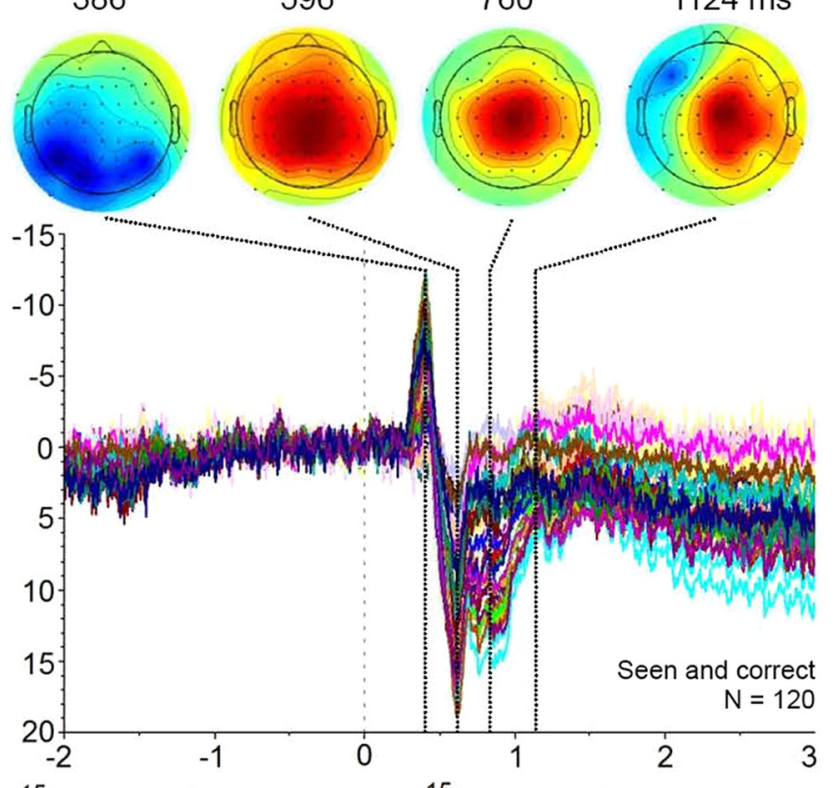

$-15$

$-10$

-10
-5

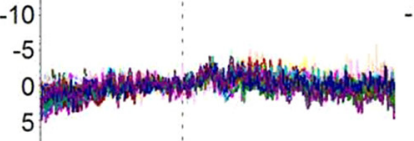

Unnseen and correct 10

\begin{tabular}{r|rrr}
15 & $\mathrm{~N}=87$ & 15 \\
20 & & 20
\end{tabular}
$760 \quad 1124 \mathrm{~ms}$
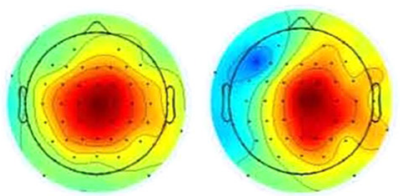

Time (sec)

Figure 10. DC-EEG data from Subj. \#12 (A) and Subj.\#13 (B). ERPs from all electrodes are shown for three conditions: Seen and correct (middle row), Unseen and correct and Unseen and incorrect (bottom row). The numbers of trials included in each condition are indicated in the figure. The scalp topography of voltage distribution at selected time points for the Seen and correct condition are shown in the top row.

after the effects of objective performance and confidence were both controlled. In the time domain, this activity lasts for up to $2 \mathrm{~s}$ after the onset of a brief stimulus ( $<67 \mathrm{~ms}$ long). In the frequency domain, the phase and power of the lowest frequencies $(<5 \mathrm{~Hz})$ correlate with subjective awareness. In contrast, objective performance and confidence are both contributed by relatively transient brain activity. These results provide strong support for the SCP hypothesis on conscious processing ( $\mathrm{He}$ and Raichle, 2009 b). Last, source modeling suggests that widespread frontoparietal and temporal cortical areas contribute to the SCPs underlying subjective awareness.

\section{Connection to previous literature on conscious perception}

Our results are consistent with the global neuronal workspace (GNW) theory of consciousness in terms of emphasizing late ( $>200 \mathrm{~ms}$ ) activity and contributions from widespread brain areas (Dehaene and Changeux, 2011). Our findings differ from the GNW framework in several aspects. First, experimental work supporting the GNW theory has emphasized brain activity in the time window of 200-600 ms (Sergent et al., 2005; Del Cul et al., 2007), while the long-lasting activity we observed persisted for $\sim 2 \mathrm{~s}$ after stimulus offset. This is potentially due to the fact that in these prior studies, a high-pass filter at around $0.5 \mathrm{~Hz}$ was applied (as opposed to $0.05 \mathrm{~Hz}$ used in the current study), such that the very slow activity was lost. Second, studies under the GNW framework have generally interpreted the late EEG activity as part of the P3b potential (Sergent et al., 2005; Del Cul et al., 2007; Lamy et al., 2009). Experimentally, this is consistent with the slow positive potential in seen trials observed in our EEG recordings (Fig. 10). However, our interpretation of these results departs from the GNW theory. We think that the slow positive potential and the related P3b potential are part of the SCP family, indicating a reduction in cortical excitability (Birbaumer and Elbert, 1988; Deecke and Lang, 1988; He and Raichle, 2009a). Thus, instead of "global ignition" or "broadcasting," we believe that these data reveal global inhibition related to the updating of conscious content/working memory and simultaneous inhibition of irrelevant information when a particular stimulus reaches conscious awareness (Birbaumer and Elbert, 1988).

Our results showing that SCPs correlate with conscious perception are consistent with an earlier study reporting that elevated neuronal firing in the medial temporal lobe can persist for $\sim 2 \mathrm{~s}$ after the recognition of a brief stimulus in a visual backward masking paradigm (Quiroga et al., 2008). Such long-lasting brain activity underlying conscious perception provides a potential mechanism for the well documented effect that conscious experience can be modulated up to hundreds of milliseconds after stimulus offset or motor output by external events, as in flash-lag illusion (Eagleman and Sejnowski, 2000), poststimulus cortical stimulation (Libet, 


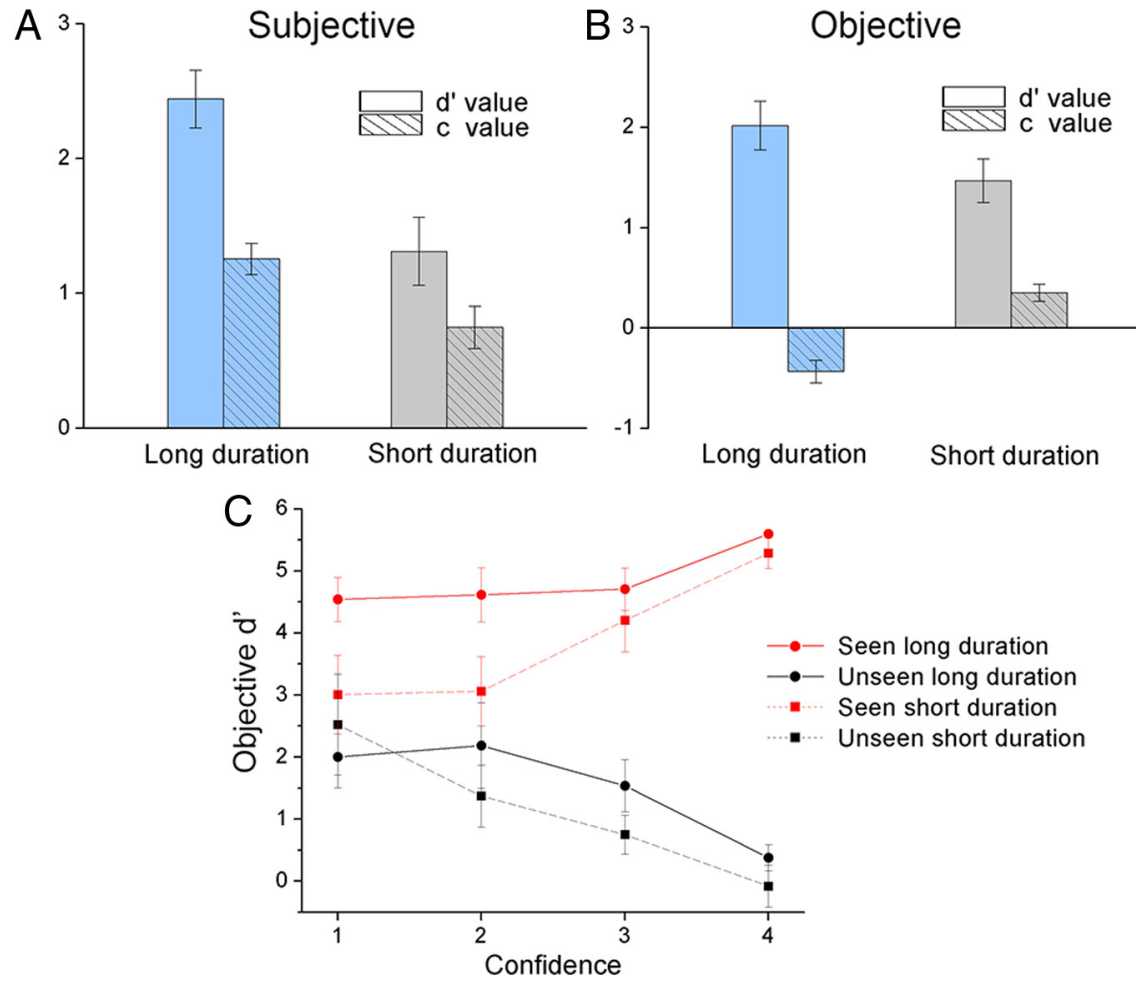

Figure 11. Behavioral control for the duration of poststimulus blank period. Behavioral data from the MEG sessions (Long duration, same as data used in Fig. 1) and from behavioral testing in an additional cohort of 11 subjects using a $200 \mathrm{~ms}$ p pststimulus blank period (Short duration) are analyzed according to signal-detection theory. $A$, Detection $d^{\prime}$ and criterion c for subjective awareness. $\boldsymbol{B}$, Discrimination $d^{\prime}$ and criterion c for objective performance. $\boldsymbol{C}$, Discrimination $d^{\prime}$ under different states of subjective awareness (seen vs unseen) and confidence level. In all graphs, error bars indicate SEM across subjects.

1982) or attentional cueing (Sergent et al., 2013), and postmovement transcranial magnetic stimulation (Lau et al., 2007).

A previous study reported that the phase of spontaneous SCP recorded by EEG over occipital cortex modulates conscious visual threshold, such that liminal stimuli presented on the negative SCP shift were more likely to be perceived (Devrim et al., 1999). This result is consistent with a large literature showing that the negative shift of SCPs indexes increased cortical excitability (Rockstroh et al., 1989; Birbaumer et al., 1990). At present the phase relationship between SCPs recorded by MEG and those recorded by EEG remains unknown. We observed that across many sensors phase $\frac{\pi}{4}$ and $-\frac{3 \pi}{4}$ of slow $(<1 \mathrm{~Hz})$ MEG activity at stimulus onset predicted a higher probability of subjectively perceiving the stimulus (Fig. 6). Phase $\frac{\pi}{4}$ and $-\frac{3 \pi}{4}$ are opposite phases to each other, consistent with the fact that MEG sensors located on opposite sides of a dipole record activities that are mirror images of each other with opposite signs. Future simultaneous EEG-MEG studies will be needed to elucidate the phase relationship between EEG- and MEG- recorded SCPs.

A recent study using a whisker stimulus detection task in rodents found more depolarized membrane potentials in primary sensory neurons at 100-300 ms following stimulus onset during hit than miss trials (Sachidhanandam et al., 2013). This result corresponds well with our EEG finding of a negative potential at $\sim 300$ ms over posterior visual regions (Fig. 10). Nonetheless, whole-brain EEG allowed us to observe the ensuing widespread positive potential over frontoparietal cortices. In addition, sub- jective report in human subjects avoids the ambiguity of using detection performance to infer the state of subjective perception.

Increased power in the gamma frequency range was previously reported to correlate with conscious perception (Fisch et al., 2009; Gaillard et al., 2009; Wyart and Tallon-Baudry, 2009). In contrast, we found gamma frequency power to correlate with objective performance but not subjective awareness (Fig. 7). A major distinction between the current work and these previous studies is that we controlled for objective performance and confidence. Consistent with our finding, a recent study found localized gamma frequency power to be correlated with sensory evidence but not conscious perception (Aru et al., 2012b).

\section{Connection to previous literature on metacognitive confidence}

We found that after the effects of subjective awareness and objective performance were controlled, early ( $200 \mathrm{~ms})$ MEG activity in the dorsal parietal cortex and late $(\sim 2.5 \mathrm{~s})$ MEG activity in the anterior prefrontal cortex both correlated with subjects' confidence levels (Fig. 8). These results are, respectively, consistent with prior primate neurophysiology (Kiani and Shadlen, 2009) and human functional/anatomical MRI (De Martino et al., 2013; McCurdy et al., 2013) studies. Our findings suggest that the different brain regions uncovered by neurophysiology and functional MRI might simply reflect the difference in temporal sensitivities of these methods.

\section{Mechanisms of SCPs}

Early neurophysiological studies suggested: "Simultaneous recording of membrane potentials, extracellular and intracellular recording from apical dendrites, and field potentials clearly demonstrate long-lasting EPSPs at the apical dendrites as the main factor underlying negative SCPs" (Birbaumer et al., 1990). However, to date the source of these long-lasting EPSPs remains unclear. Nonetheless, there are several plausible and not mutually exclusive candidates. First, recurrent excitatory neuronal networks may constitute a prominent source of long-lasting EPSPs (Major and Tank, 2004; Wong and Wang, 2006; Chaudhuri et al., 2014). Second, long-lasting cellular mechanisms such as calcium spikes (Larkum, 2013), metabotropic receptors (Zhang and Séguéla, 2010), and the endocannabanoid pathway (Carter and Wang, 2007) could potentially produce long-lasting EPSPs in the SCP timescale. Third, it has been long conjectured that neural modulations such as acetylcholine might play a role in generating SCPs (Birbaumer et al., 1990). A recent study demonstrating that cholinergic inputs to layer I could selectively disinhibit layer II/III pyramidal neurons lends substantial credence to this idea (Letzkus et al., 2011). Future experimental investigations should test and refine these predictions. A promising avenue is to combine DC recordings of field potentials (Kahn et al., 2013; Pan et al., 
2013) with pharmacological manipulations or stimulation of specific cell types in different cortical layers.

\section{Caveats and future questions}

Our results suggest that the waveform, phase, and power of slow MEG activity correlate with the state of subjective awareness from trial to trial, after objective performance and confidence were both controlled. These results provide strong support for the SCP hypothesis on conscious processing (He and Raichle, 2009b). Nonetheless, it is important to clarify that not all SCPs are directly related to conscious processing. For example, unconscious modulation of brain excitability can also manifest as SCPs (Libet et al., 1983; Elbert, 1990). This is not in conflict with the SCP hypothesis on conscious processing- as we stated in the original paper (He and Raichle, 2009b), "these results, however, do not suggest that the negative SCP, whenever it appears, is an index of conscious awareness." A crucial goal for future research is to elucidate the difference in spatiotemporal patterns of the SCPs related to unconscious versus conscious processing.

Second, the relationship between our results and various forms of memory should be explored in future experiments. It is unlikely that visual persistence or iconic memory confounds our results. Visual persistence lasts up to several hundred milliseconds and iconic memory up to a second-both are much shorter than our SCP findings (Sperling, 1960; Coltheart, 1980). Moreover, both visual persistence and iconic memory are strongest right after stimulus offset and decay in a monotonic fashion; in contrast, we found that SCPs correlated with subjective awareness reach their peak at $\sim 500 \mathrm{~ms}$ and decay thereafter (Figs. 2C, 3 and 10). Nonetheless, the potential roles of fragile visual shortterm memory (FM) and working memory (WM) should be further investigated in future studies. Existing evidence suggests that FM is a form of location- and object- specific short-term memory store that is likely mediated by higher order visual areas such as V4 and inferotemporal cortex (Sligte et al., 2009; Pinto et al., 2013). As such, neural processes underlying FM might manifest in the negative SCP potential peaking at $300-400 \mathrm{~ms}$ localized to posterior visual regions that were observed in our EEG experiment (Fig. 10). Moreover, as discussed earlier, the widespread cortical inhibition thereafter, indicated by slow positive potentials at $>500 \mathrm{~ms}$ (Fig. 10), might be related to the updating of WM when a stimulus reaches conscious awareness. At present, it remains unclear whether these-conscious access, FM, and WM update-are separable processes (see discussions in Sligte et al., 2008). In addition, it is possible that delaying responses in our paradigm introduced a sustained WM component (Vogel and Machizawa, 2004); future studies dissociating these processes will be valuable.

Last, the separation of the NCC from NCC-pr and NCC-co in our study is likely incomplete. Our analyses were able to disentangle these phenomena to the extent that objective performance captures NCC-pr and metacognitive confidence captures NCCco. However, there are likely NCC-pr and NCC-co processes not captured by these measures. For example, it has long been postulated that conscious perception could lead to verbal report or the formation of long-term memory (Baars, 1988). Although our paradigm did not involve overt verbal report, and the effect of long-term memory was likely mitigated by repeated presentations of a minimal stimulus, at present it is not possible to rule out latent processes as such. These caveats notwithstanding, the current study constitutes a significant step forward in the ongoing effort to disentangle conscious from unconscious brain processes and distill the NCC from NCC-pr and NCC-co.

\section{References}

Aru J, Bachmann T, Singer W, Melloni L (2012a) Distilling the neural correlates of consciousness. Neurosci Biobehav Rev 36:737-746. CrossRef Medline

Aru J, Axmacher N, Do Lam AT, Fell J, Elger CE, Singer W, Melloni L (2012b) Local category-specific gamma band responses in the visual cortex do not reflect conscious perception. J Neurosci 32:14909-14914. CrossRef Medline

Baars BJ (1988) A cognitive theory of consciousness. New York: Cambridge UP.

Bachmann T (2009) Finding ERP-signatures of target awareness: puzzle persists because of experimental co-variation of the objective and subjective variables. Conscious Cogn 18:804-808; discussion 809-810. CrossRef Medline

Bell AJ, Sejnowski TJ (1995) An information-maximization approach to blind separation and blind deconvolution. Neural Comput 7:1129-1159. CrossRef Medline

Berens P (2009) CircStat: a MATLAB toolbox for circular statistics. J Stat Software 31:1-21.

Birbaumer N, Elbert T (1988) P3: byproduct of a byproduct. Behav Brain Sci 11:375-377.

Birbaumer N, Elbert T, Canavan AG, Rockstroh B (1990) Slow potentials of the cerebral cortex and behavior. Physiol Rev 70:1-41. Medline

Brookes MJ, Gibson AM, Hall SD, Furlong PL, Barnes GR, Hillebrand A, Singh KD, Holliday IE, Francis ST, Morris PG (2005) GLMbeamformer method demonstrates stationary field, alpha ERD and gamma ERS co-localisation with fMRI BOLD response in visual cortex. Neuroimage 26:302-308. CrossRef Medline

Brookes MJ, Hale JR, Zumer JM, Stevenson CM, Francis ST, Barnes GR, Owen JP, Morris PG, Nagarajan SS (2011) Measuring functional connectivity using MEG: methodology and comparison with fcMRI. Neuroimage 56:1082-1104. CrossRef Medline

Busch NA, Dubois J, VanRullen R (2009) The phase of ongoing EEG oscillations predicts visual perception. J Neurosci 29:7869-7876. CrossRef Medline

Carter E, Wang XJ (2007) Cannabinoid-mediated disinhibition and working memory: dynamical interplay of multiple feedback mechanisms in a continuous attractor model of prefrontal cortex. Cereb Cortex 17 [Suppl 1]:i16i26. CrossRef Medline

Chaudhuri R, He BJ, Wang XJ (2014) The temporal structure of a random network near criticality and human ECoG dynamics. COSYNE, Salt Lake City, Abstract 194.

Coltheart M (1980) Iconic memory and visible persistence. Perception and psychophysics 27:183-228. CrossRef Medline

Dale AM, Sereno MI (1993) Improved localization of cortical activity by combining EEG and MEG with MRI cortical surface reconstruction: a linear approach. J Cogn Neurosci 5:162-176. CrossRef Medline

Deecke L, Lang W (1988) P300 as the resolution of negative cortical DC shifts. Behav Brain Res 11:379-381.

de Graaf TA, Hsieh PJ, Sack AT (2012) The 'correlates' in neural correlates of consciousness. Neurosci Biobehav Rev 36:191-197. CrossRef Medline

Dehaene S, Changeux JP (2011) Experimental and theoretical approaches to conscious processing. Neuron 70:200-227. CrossRef Medline

Dehaene S, Changeux JP, Naccache L, Sackur J, Sergent C (2006) Conscious, preconscious, and subliminal processing: a testable taxonomy. Trends Cogn Sci 10:204-211. CrossRef Medline

Del Cul A, Baillet S, Dehaene S (2007) Brain dynamics underlying the nonlinear threshold for access to consciousness. PLoS Biol 5:e260. CrossRef Medline

Del Cul A, Dehaene S, Reyes P, Bravo E, Slachevsky A (2009) Causal role of prefrontal cortex in the threshold for access to consciousness. Brain 132: 2531-2540. CrossRef Medline

De Martino B, Fleming SM, Garrett N, Dolan RJ (2013) Confidence in value-based choice. Nat Neurosci 16:105-110. CrossRef Medline

Devrim M, Demiralp T, Kurt A, Yücesir I (1999) Slow cortical potential shifts modulate the sensory threshold in human visual system. Neurosci Lett 270:17-20. CrossRef Medline

Eagleman DM, Sejnowski TJ (2000) Motion integration and postdiction in visual awareness. Science 287:2036-2038. CrossRef Medline

Elbert T (1990) Slow cortical potentials reflect the regulation of cortical excitability. In: Slow potential changes in the human brain (McCallum WC, ed), pp 235-251. New York: Plenum. 
Fisch L, Privman E, Ramot M, Harel M, Nir Y, Kipervasser S, Andelman F, Neufeld MY, Kramer U, Fried I, Malach R (2009) Neural "ignition": enhanced activation linked to perceptual awareness in human ventral stream visual cortex. Neuron 64:562-574. CrossRef Medline

Fleming SM, Weil RS, Nagy Z, Dolan RJ, Rees G (2010) Relating introspective accuracy to individual differences in brain structure. Science 329: 1541-1543. CrossRef Medline

Gaillard R, Dehaene S, Adam C, Clémenceau S, Hasboun D, Baulac M, Cohen L, Naccache L (2009) Converging intracranial markers of conscious access. PLoS Biol 7:e61. CrossRef Medline

Green DM, Swets JA (1966) Signal detection theory and psychophysics. New York: Wiley.

Hämäläinen MS (2005) MNE software user's guide: NMR Center. Harvard University, Massachusetts General Hospital.

He BJ (2013) Spontaneous and task-evoked brain activity negatively interact. J Neurosci 33:4672-4682. CrossRef Medline

He BJ, Raichle ME (2009a) Response to Koch: elaborations on the SCP hypothesis. Trends Cogn Sci 13:368-369. CrossRef Medline

He BJ, Raichle ME (2009b) The fMRI signal, slow cortical potential and consciousness. Trends Cogn Sci 13:302-309. CrossRef Medline

He BJ, Zempel JM (2013) Average is optimal: an inverted-U relationship between trial-to-trial brain activity and behavioral performance. PLoS Comput Biol 9:e1003348. CrossRef Medline

He BJ, Snyder AZ, Zempel JM, Smyth MD, Raichle ME (2008) Electrophysiological correlates of the brain's intrinsic large-scale functional architecture. Proc Natl Acad Sci U S A 105:16039-16044. CrossRef Medline

Hesselmann G, Hebart M, Malach R (2011) Differential BOLD activity associated with subjective and objective reports during "blindsight" in normal observers. J Neurosci 31:12936-12944. CrossRef Medline

Jokisch D, Jensen O (2007) Modulation of gamma and alpha activity during a working memory task engaging the dorsal or ventral stream. J Neurosci 27:3244-3251. CrossRef Medline

Kahn I, Knoblich U, Desai M, Bernstein J, Graybiel AM, Boyden ES, Buckner RL, Moore CI (2013) Optogenetic drive of neocortical pyramidal neurons generates fMRI signals that are correlated with spiking activity. Brain Res 1511:33-45. CrossRef Medline

Kiani R, Shadlen MN (2009) Representation of confidence associated with a decision by neurons in the parietal cortex. Science 324:759-764. CrossRef Medline

Lachaux JP, Rodriguez E, Martinerie J, Varela FJ (1999) Measuring phase synchrony in brain signals. Hum Brain Mapp 8:194-208. CrossRef Medline

Lamme VA (2003) Why visual attention and awareness are different. Trends Cogn Sci 7:12-18. CrossRef Medline

Lamy D, Salti M, Bar-Haim Y (2009) Neural correlates of subjective awareness and unconscious processing: an ERP study. J Cogn Neurosci 21: 1435-1446. CrossRef Medline

Larkum M (2013) A cellular mechanism for cortical associations: an organizing principle for the cerebral cortex. Trends Neurosci 36:141-151. CrossRef Medline

Lau HC, Passingham RE (2006) Relative blindsight in normal observers and the neural correlate of visual consciousness. Proc Natl Acad Sci U S A 103:18763-18768. CrossRef Medline

Lau HC, Rogers RD, Passingham RE (2007) Manipulating the experienced onset of intention after action execution. J Cogn Neurosci 19:81-90. CrossRef Medline

Leistner S, Sander T, Burghoff M, Curio G, Trahms L, Mackert BM (2007) Combined MEG and EEG methodology for non-invasive recording of infraslow activity in the human cortex. Clin Neurophysiol 118:27742780. CrossRef Medline

Letzkus JJ, Wolff SB, Meyer EM, Tovote P, Courtin J, Herry C, Lüthi A (2011) A disinhibitory microcircuit for associative fear learning in the auditory cortex. Nature 480:331-335. CrossRef Medline

Levitt H (1971) Transformed up-down methods in psychoacoustics. J Acoust Soc Am 49[Suppl 2]:467. CrossRef Medline

Libet B (1982) Brain stimulation in the study of neuronal functions for conscious sensory experiences. Hum Neurobiol 1:235-242. Medline

Libet B, Gleason CA, Wright EW, Pearl DK (1983) Time of conscious intention to act in relation to onset of cerebral activity (readiness-potential). The unconscious initiation of a freely voluntary act. Brain 106:623-642. CrossRef Medline

Major G, Tank D (2004) Persistent neural activity: prevalence and mechanisms. Curr Opin Neurobiol 14:675-684. CrossRef Medline
Mathewson KE, Gratton G, Fabiani M, Beck DM, Ro T (2009) To see or not to see: prestimulus alpha phase predicts visual awareness. J Neurosci 29: 2725-2732. CrossRef Medline

McCurdy LY, Maniscalco B, Metcalfe J, Liu KY, de Lange FP, Lau H (2013) Anatomical coupling between distinct metacognitive systems for memory and visual perception. J Neurosci 33:1897-1906. CrossRef Medline

Medendorp WP, Kramer GF, Jensen O, Oostenveld R, Schoffelen JM, Fries P (2007) Oscillatory activity in human parietal and occipital cortex shows hemispheric lateralization and memory effects in a delayed double-step saccade task. Cereb Cortex 17:2364-2374. CrossRef Medline

Mitzdorf U (1985) Current source-density method and application in cat cerebral cortex: investigation of evoked potentials and EEG phenomena. Physiol Rev 65:37-100. Medline

Nichols TE, Holmes AP (2002) Nonparametric permutation tests for functional neuroimaging: a primer with examples. Hum Brain Mapp 15:1-25. CrossRef Medline

Pan WJ, Thompson GJ, Magnuson ME, Jaeger D, Keilholz S (2013) Infraslow LFP correlates to resting-state fMRI BOLD signals. Neuroimage 74:288-297. CrossRef Medline

Pinto Y, Sligte IG, Shapiro KL, Lamme VA (2013) Fragile visual short-term memory is an object-based and location-specific store. Psychon Bull Rev 20:732-739. CrossRef Medline

Quiroga RQ, Mukamel R, Isham EA, Malach R, Fried I (2008) Human single-neuron responses at the threshold of conscious recognition. Proc Natl Acad Sci U S A 105:3599-3604. CrossRef Medline

Rockstroh B, Elbert T, Canavan A, Lutzenberger W, Birbaumer N (1989) Slow brain potentials and behaviour, Ed 2. München, Germany: Urban and Schwarzenberg.

Rounis E, Maniscalco B, Rothwell JC, Passingham RE, Lau H (2010) Thetaburst transcranial magnetic stimulation to the prefrontal cortex impairs metacognitive visual awareness. Cogn Neurosci 1:165-175. CrossRef Medline

Sachidhanandam S, Sreenivasan V, Kyriakatos A, Kremer Y, Petersen CC (2013) Membrane potential correlates of sensory perception in mouse barrel cortex. Nat Neurosci 16:1671-1677. CrossRef Medline

Sergent C, Baillet S, Dehaene S (2005) Timing of the brain events underlying access to consciousness during the attentional blink. Nat Neurosci 8:1391-1400. CrossRef Medline

Sergent C, Wyart V, Babo-Rebelo M, Cohen L, Naccache L, Tallon-Baudry C (2013) Cueing attention after the stimulus is gone can retrospectively trigger conscious perception. Curr Biol 23:150-155. CrossRef Medline

Sligte IG, Scholte HS, Lamme VA (2008) Are there multiple visual shortterm memory stores? PLoS One 3:e1699. CrossRef Medline

Sligte IG, Scholte HS, Lamme VA (2009) V4 activity predicts the strength of visual short-term memory representations. J Neurosci 29:7432-7438. CrossRef Medline

Sperling G (1960) The information available in brief visual presentations. Psychol Monogr Gen Appl 74:1-29. CrossRef

Tallon-Baudry C, Bertrand O, Delpuech C, Pernier J (1996) Stimulus specificity of phase-locked and non-phase-locked $40 \mathrm{~Hz}$ visual responses in human. J Neurosci 16:4240-4249. Medline

Vogel EK, Machizawa MG (2004) Neural activity predicts individual differences in visual working memory capacity. Nature 428:748-751. CrossRef Medline

Watthanacheewakul L (2010) Analysis of variance with Weibull data. In: Proceedings of the International Multiconference of Engineers and Computer Scientists. Hong Kong: IMECS.

Weiskrantz L (2004) Roots of blindsight. Prog Brain Res 144:229-241. Medline

Wong KF, Wang XJ (2006) A recurrent network mechanism of time integration in perceptual decisions. J Neurosci 26:1314-1328. CrossRef Medline

Wyart V, Tallon-Baudry C (2008) Neural dissociation between visual awareness and spatial attention. J Neurosci 28:2667-2679. CrossRef Medline

Wyart V, Tallon-Baudry C (2009) How ongoing fluctuations in human visual cortex predict perceptual awareness: baseline shift versus decision bias. J Neurosci 29:8715-8725. CrossRef Medline

Yao J, Dewald JP (2005) Evaluation of different cortical source localization methods using simulated and experimental EEG data. Neuroimage 25: 369-382. CrossRef Medline

Zhang Z, Séguéla P (2010) Metabotropic induction of persistent activity in layers II/III of anterior cingulate cortex. Cereb Cortex 20:2948-2957. CrossRef Medline 